\title{
Oka and Ako Ideal Families in Commutative Rings
}

\author{
T. Y. Lam and Manuel L. Reyes
}

\begin{abstract}
In our earlier study [LR] of prime ideal principles in commutative rings, we have introduced the notion of Oka and Ako families of ideals, along with their "strong analogues". The logical hierarchy between these ideal families (and the classically well-known monoidal families of ideals) was partly worked out in $[\mathrm{LR}]$ over general commutative rings. In the present paper, we amplify this study by developing further hierarchical relationships among these families over special classes of rings, and also by finding concrete examples of ideal families to show that certain such relationships cannot be reversed or improved. The special types of commutative rings studied here include von Neumann regular rings and integral domains (for instance Dedekind domains and valuation domains).
\end{abstract}

\section{Introduction}

In commutative algebra, there are many theorems of the "maximal implies prime" variety. To begin with, maximal ideals are prime, and more generally, an ideal maximal with respect to being disjoint from a given multiplicative set $S$ is prime [Ka: p.1]. Another well-known example is that an ideal maximal with respect to not being finitely generated is prime [Co]. Most of these theorems can be formulated in the language of ideal systems as follows: for a specific family $\mathcal{F}$ of ideals in a commutative ring $R$ (with $R \in \mathcal{F}$ ), an ideal maximal with respect to not being in $\mathcal{F}$ is prime. In the examples given above, $\mathcal{F}$ can be taken to be, respectively, the family of ideals intersecting $S$, and the family of f.g. (finitely generated) ideals of $R$.

In the commutative algebra literature (up to 2007), the large number of "maximal implies prime" theorems were proved on a case-by-case basis, with each one requiring a separate analysis that looks quite different from one case to another. To remedy this situation, we introduced a general Prime Ideal Principle in [LR: Th. 2.4] to bring such theorems under one roof. Crucial to the formulation of the Prime Ideal Principle are the notions of Oka and Ako families of ideals in a commutative ring, which we recall as follows.

1991 Mathematics Subject Classification. Primary 13A15, 13E05; Secondary 13F05, 13F30.

Key words and phrases. Commutative rings, Prime Ideal Principle, multiplicative theory of ideals, ideal systems, monoidal systems, semifilters, Oka and Ako families, conormal modules, von Neumann regular rings, Dedekind domains, and valuation domains. 
Definition 1.1. An ideal family $\mathcal{F}$ in a ring $R$ with $R \in \mathcal{F}$ is said to be an Oka family (resp. strongly Oka family) if, for $a \in R$ and $I, A \triangleleft R,(I, a),(I: a) \in \mathcal{F}$ $\Longrightarrow I \in \mathcal{F}$ (resp. $(I, A),(I: A) \in \mathcal{F} \Longrightarrow I \in \mathcal{F})$.

Definition 1.2. An ideal family $\mathcal{F}$ in a ring $R$ with $R \in \mathcal{F}$ is said to be an $A k o$ family (resp. strongly Ako family) if, for $a, b \in R$ and $I, B \triangleleft R,(I, a),(I, b) \in \mathcal{F}$ $\Longrightarrow(I, a b) \in \mathcal{F}$ (resp. $(I, a),(I, B) \in \mathcal{F} \Longrightarrow(I, a B) \in \mathcal{F})$.

Of course, if $R$ is a PIR (principal ideal ring), then "Oka" and "Strongly Oka" are the same concepts, and so are "Ako" and "Strongly Ako". For general rings, however, the strong versions can be expected to be more restrictive. The notion of Oka families was inspired by a classical function-theoretic result of Oka [Ok: Corollaire 2, p. 209], which amounts to the fact that f.g. ideals form an Oka family in any commutative ring. The name "Ako family" in (1.2) was simply coined as a light-hearted parody of an Oka family. The main result in $[\mathrm{LR}]$ is the following simple statement.

(1.3) Prime Ideal Principle (P.I.P.). If $\mathcal{F}$ is an Oka family or an Ako family, then any ideal maximal w.r.t. not being in $\mathcal{F}$ is prime.

To the best of our knowledge, this P.I.P. seemed to have covered most theorems in the literature of the "maximal implies prime" variety. Thus, the P.I.P. uniformizes the proofs of all such results into checking the Oka or Ako property of a suitable system of ideals. On the other hand, the degree of generality attained by using such a unified approach led to many more results of a similar nature, as in $\S \S 3-5$ of $[\mathrm{LR}]$.

The notion of Oka, Ako families and their strong analogues may also be thought of as generalizations of the classically well-known notion of a monoidal filter. To recall the relevant definitions, let $\mathcal{F}$ be a family of ideals in $R$ with $R \in \mathcal{F}$. We say

(1) $\mathcal{F}$ is a semifilter if, for all $I, J \triangleleft R, I \supseteq J \in \mathcal{F} \Rightarrow I \in \mathcal{F}$;

(2) $\mathcal{F}$ is a filter if it is a semifilter and $A, B \in \mathcal{F} \Rightarrow A \cap B \in \mathcal{F}$;

(3) $\mathcal{F}$ is monoidal if $A, B \in \mathcal{F} \Rightarrow A B \in \mathcal{F}$; that is, $\mathcal{F}$ is a submonoid of the monoid of all ideals of $R$ under multiplication; and

(4) $\mathcal{F}$ is $\frac{1}{2}$-monoidal if $(a), B \in \mathcal{F} \Rightarrow a B \in \mathcal{F}$.

It is easy to see that "monoidal filter" is the same as "monoidal semifilter", and that a monoidal filter is always (strongly) Oka and Ako. However, monoidal filters are very restrictive, so a P.I.P. formulated for them alone would not be applicable to many ideal families. This was exactly the reason for introducing the broader notion of the Oka and Ako families of ideals, for which P.I.P. turned out to hold already.

In [LR: (2.7)], we have made an initial study of the logical dependence relations between the various ideal families mentioned in the last paragraph. For the convenience of the reader, a recapitulation of this result is given in (2.6) below (see, especially, the chart (2.7)). However, no attempts were made in [LR] to show how exhaustive these relations were, and only a few examples were given to "separate" the various families. It was shown, for instance, that Oka families need not be Ako, but the question whether Ako families are Oka was left open. This is a significant 
question, since an affirmative answer for it would have implied that all common "maximal $\Rightarrow$ prime" theorems could have been deduced from the P.I.P. for Oka families alone.

The goal of the present paper is to study more systematically the hierarchical relationships between "Oka", "Ako", their strong analogues, and some other properties $\left(\mathrm{P}_{i}\right)$ introduced in [LR: $\left.(2.7)\right]$. These properties $\left(\mathrm{P}_{i}\right)(i=1,2,3)$ are recalled in $\S 2$, where $\left(\mathrm{P}_{2}\right)$ and $\left(\mathrm{P}_{3}\right)$ are given more streamlined descriptions via the supporting "squeezing properties" $(\mathrm{Sq})$ and $(\mathrm{Sq})^{*}$. The logical dependence relations are greatly simplified if we consider semifilter families $\mathcal{F}$. For instance, for such $\mathcal{F}$ in a noetherian ring, all of the above properties (besides $(\mathrm{Sq})$ and $(\mathrm{Sq})^{*}$ ) turn out to be equivalent to the Prime Ideal Principle (Thm. (3.2)). On the other hand, studying these properties "in microcosm" - in the case of a two-member family $\mathcal{F}=\{R, A\}$, we can reinterpret each of them in terms of natural ideal-theoretic properties on $A$ (Thms. (3.4), (3.5), and (3.6)). This study leads to several classes of ideals of independent interest (Ako, Oka, and strongly Oka ideals), and shows that, already in the case $|\mathcal{F}|=2$, all but one of the seven basic implications in the chart of logical dependence (2.7) are irreversible. These results are presented in $\S 3$.

The remaining sections $\S \S 4-7$ of the paper are devoted to the development of further relationships between the various ideal family properties over special classes of rings; e.g. von Neumann regular rings, Dedekind domains, and valuation domains. These relationships are not always easy to predict, as some of them seem a little more "hidden" than others. They also vary rather substantially from one class of rings to another, and from one kind of ideal family to another. Without trying to be systematic or exhaustive, we'll just summarize some of the more representative results below.

- The Oka family of all f.g. ideals in a ring need not be strongly Oka.

- Over von Neumann rings, $\left(\mathrm{P}_{2}\right),\left(\mathrm{P}_{3}\right)$ are equivalent to the monoidal property, and each of "Oka" and "Ako" is equivalent to a suitable partial monoidal property.

- Over von Neumann rings, Dedekind domains, and 1-dimensional valuation domains (and in the case $|\mathcal{F}|=2$ as well), Ako families are Oka. However, this is not the case over higher dimensional valuation domains.

- For arbitrary families over a Dedekind ring, Oka $\Leftrightarrow$ Strongly Oka $\Leftrightarrow$ Monoidal, and Ako $\Leftrightarrow$ Strongly Ako $\Leftrightarrow\left(\mathrm{P}_{3}\right)$. The latter equivalences also hold for any family of principal ideals over any ring, but not for general families.

- Over integral domains (and for arbitrary families), Oka $\Rightarrow \frac{1}{2}$-Monoidal, and the converse holds over any valuation domain. However, the same results do not hold over von Neumann regular rings.

- Over a noetherian domain, even strongly Oka families need not be monoidal or Ako, and Oka families need not be strongly Oka.

- Over a valuation domain, Oka and Ako families as well as $\left(\mathrm{P}_{3}\right)$ families are given simpler characterizations, and they are explicitly determined. The logical dependence chart (2.7) is again shown to be best possible for valuation domains.

It may be mentioned, as a kind of philosophical remark, that the work in this paper (and in its predecessor [LR]) exploits much more the multiplicative structure of ideals than their additive structure. It would perhaps be appropriate, therefore, 
to view this work as a part of the multiplicative theory of ideals in rings, started by Dedekind, Noether, Krull [Kr], Lorenzen [Lo], continued by many others, and exposited in the books of Jaffard [Ja], Gilmer [Gi], Larsen-McCarthy [LM], and Halter-Koch [HK]; see also [BG]. The various notions and techniques on ideal systems introduced in $[\mathrm{LR}]$ and the present paper seem to provide some new avenues along which to study the multiplicative structure of ideals in commutative rings. To encourage further work along these lines, a few open questions are mentioned in $(2.8),(5.6),(5.10)$, and (6.5).

In this paper, all rings considered are commutative (with identity), and all ideal families $\mathcal{F}$ in a ring $R$ are assumed to contain $R$. The notation $I \triangleleft R$ means that $I$ is an ideal in $R$, and $(J)$ denotes the ideal generated by a set $J \subseteq R$. Other commutative algebra notations and terminology follow those in Kaplansky's text $[\mathrm{Ka}]$, while the main results and examples in $[\mathrm{LR}]$ will be invoked rather freely throughout.

\section{Families of Principal and Finitely Generated Ideals}

We start by recalling from [LR: $\S 2]$ the definitions of the three properties $\left(\mathrm{P}_{i}\right)$ for ideal families $\mathcal{F}$ in any ring $R$ (with $R \in \mathcal{F}$ ). Here, we'll give a more systematic introduction to $\left(\mathrm{P}_{2}\right)$ and $\left(\mathrm{P}_{3}\right)$ by focusing on two (apparently dual) "squeezing properties" called (Sq) and (Sq)*. These are defined in the following result.

Proposition 2.1. For any ideal family $\mathcal{F}$ in $R$, the following properties for $n \geq 2$ are mutually equivalent (and will henceforth be denoted by $(\mathrm{Sq})$ ):

$(\mathrm{Sq})_{n} \quad$ For $I, J \triangleleft R$ such that $J^{n} \subseteq I \subseteq J, J \in \mathcal{F} \Rightarrow I \in \mathcal{F}$.

The following properties for $n \geq 2$ are also mutually equivalent (and will henceforth be denoted by $\left.(\mathrm{Sq})^{*}\right)$ :

For $I, J \triangleleft R$ such that $J^{n} \subseteq I \subseteq J, I \in \mathcal{F} \Rightarrow J \in \mathcal{F}$.

Proof. For the first part, we need only prove $(\mathrm{Sq})_{2} \Rightarrow(\mathrm{Sq})_{n}$ (for any $n \geq 2$ ). Assume that $J^{n} \subseteq I \subseteq J$ with $J \in \mathcal{F}$ but $I \notin \mathcal{F}$. Noting that $I+J=J \in \mathcal{F}$ but $I+J^{n}=I \notin \mathcal{F}$, we can choose a largest $k \geq 1$ such that $I+J^{k} \in \mathcal{F}$. Then

$$
\left(I+J^{k}\right)^{2} \subseteq I+J^{k+1} \subseteq I+J^{k}
$$

implies that $I+J^{k+1} \in \mathcal{F}$ (by $(\mathrm{Sq})_{2}$ ), a contradiction.

For the second part, we need to prove $(\mathrm{Sq})_{2}^{*} \Rightarrow(\mathrm{Sq})_{n}^{*}$ (for any $n \geq 2$ ). Assume that $J^{n} \subseteq I \subseteq J$ with $I \in \mathcal{F}$ but $J \notin \mathcal{F}$. Noting that $I+J=J \notin \mathcal{F}$ but $I+J^{n}=I \in \mathcal{F}$, we can choose a largest $k \geq 1$ such that $I+J^{k} \notin \mathcal{F}$. Then (2.2) implies that $I+J^{k+1} \notin \mathcal{F}$ (by $(\mathrm{Sq})_{2}^{*}$ ), a contradiction. follows:

With the above result in place, the three properties $\left(\mathrm{P}_{i}\right)$ may be introduced as

$\left(\mathrm{P}_{1}\right) \mathcal{F}$ is a monoidal filter.

$\left(\mathrm{P}_{2}\right) \mathcal{F}$ is closed under pairwise products, and satisfies $(\mathrm{Sq})^{*}$.

$\left(\mathrm{P}_{3}\right) \mathcal{F}$ is closed under pairwise intersections, and satisfies (Sq). 
The advantage of this formulation of the definitions is that $\left(\mathrm{P}_{2}\right)$ and $\left(\mathrm{P}_{3}\right)$ appear in the same light. The following proposition shows that the above definition of $\left(\mathrm{P}_{3}\right)$ is consistent with that originally given in [LR: (2.7)].

Proposition 2.3. An ideal family $\mathcal{F}$ in $R$ satisfies $\left(\mathrm{P}_{3}\right)$ iff it has the following property

$$
\forall I, A, B \triangleleft R: \quad(I, A),(I, B) \in \mathcal{F} \Longrightarrow(I, A B) \in \mathcal{F} .
$$

In this case, $\mathcal{F}$ is always monoidal.

Proof. Suppose $\mathcal{F}$ satisfies $\left(\mathrm{P}_{3}\right)$. Given $I, A, B$ in $(2.4)$, let $A^{\prime}=(I, A) \in \mathcal{F}$, and $B^{\prime}=(I, B) \in \mathcal{F}$. Then $A^{\prime} \cap B^{\prime} \in \mathcal{F}$. Since

$$
\left(A^{\prime} \cap B^{\prime}\right)^{2} \subseteq A^{\prime} B^{\prime} \subseteq(I, A B) \subseteq A^{\prime} \cap B^{\prime},
$$

$(\mathrm{Sq})_{2}$ implies that $(I, A B) \in \mathcal{F}$. Conversely, suppose $\mathcal{F}$ satisfies (2.4). To check (Sq) 2 , consider $A^{2} \subseteq I \subseteq A$, where $A \in \mathcal{F}$. Then, by (2.4) (in the special case $A=B),(I, A)=A \in \mathcal{F} \Rightarrow I=\left(I, A^{2}\right) \in \mathcal{F}$. Finally, to check that $\mathcal{F}$ is closed under pairwise intersections, let $A, B \in \mathcal{F}$. Then $(A \cap B, A)=A \in \mathcal{F}$ and $(A \cap B, B)=B \in \mathcal{F}$ imply $A \cap B=(A \cap B, A B) \in \mathcal{F}$, by (2.4). The fact that $\mathcal{F}$ must be monoidal follows by taking $I=0$ in (2.4).

For the reader's convenience, we recall below the basic logical dependence relations developed in $[\mathrm{LR}]$ between the Oka, Ako properties, their strong analogues, and the properties $\left(\mathrm{P}_{i}\right)$. Most of the implications are straightforward, and all of them were fully proved in [LR: (2.7)].

Logical Dependence Theorem 2.6. For an ideal family $\mathcal{F}$ in $R$ with $R \in \mathcal{F}$, we have the following chart of implications:

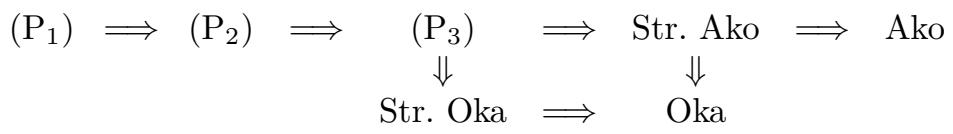

In this paper, we'll show that this chart of implications is complete, in the sense that no new implication arrows can be added to it (other than those obtainable by compositions). This statement is made, of course, for general ideal families in general rings. For a large part of this paper, we'll also consider special ideal families $(\S \S 2-3)$ and special rings ( $\S \S 4-7)$, and study additional relationships that may exist between the seven properties appearing in the chart (2.7). So far, the existence or non-existence of such additional relationships does not seem to follow any general pattern, and can be ascertained only by ad hoc considerations.

Two of the motivating examples in the study of Oka families are the families of f.g. and principal ideals in a ring $R$. Let us denote these families, respectively, by $\mathcal{F}_{\text {fg }}(R)$ and $\mathcal{F}_{\text {pr }}(R)$. Starting with the former, which is an Oka family by the classical result of Oka ([Ok: Cor.2], [Na: (3.3)]), we may already ask the following

Question 2.8. For what rings $R$ is $\mathcal{F}_{\mathrm{fg}}(R)$ strongly Oka? (In more explicit terms, these are rings for which we have a strong form of Oka's Corollaire:

- If $(I, A)$ and $(I: A)$ are f.g., then so is $I$;

or equivalently, if $I \subseteq J$, and $J,(I: J)$ are f.g., then so is $I$.) 
This is certainly the case for noetherian rings $R$, but these are not the only ones. For instance, if $R$ is a ring in which all f.g. ideals are principal, then $\mathcal{F}_{\text {fg }}(R)=$ $\mathcal{F}_{\mathrm{pr}}(R)$, and the latter is always strongly Oka (for any $R$ ), by [LR: (3.17)]. This case includes all von Neumann regular rings and all Bézout domains (in particular, all valuation domains). To produce more examples, we recall the following useful commutative algebra notions which dated back to Asano, Krull, and Mori (see, e.g. [Mo], [GM], $\left.\left[\mathrm{An}_{1}\right]\right)$.

Definition 2.9. An ideal $J \triangleleft R$ is called a multiplication ideal if every ideal $I \subseteq J$ has the form $J K$ for some $K \triangleleft R$ (or equivalently, $I=J \cdot(I: J)$ ). ${ }^{1}$ A ring $\bar{R}$ is called a multiplication ring if every $J \triangleleft R$ is a multiplication ideal.

Examples of multiplication ideals include all invertible ideals, all principal ideals, and all ideals that are generated by sets of idempotents. Thus, von Neumann regular rings, PIRs, and Dedekind rings are multiplication rings. In partial answer to (2.8), we have (2) of the following result.

Proposition 2.10. (1) If $\mathcal{F}$ is a monoidal family of multiplication ideals in a ring $R$, then $\mathcal{F}$ is strongly Oka. In particular, the family of all (f.g.) multiplication ideals in $R$ is strongly Oka. (2) If $R$ is a multiplication ring, then $\mathcal{F}_{\mathrm{fg}}(R)$ is strongly Oka.

Proof. (1) Thanks to a slight reformulation of the "strongly Oka" notion in [LR: (2.7)], it suffices to check that, if $I \subseteq J$ are ideals such that $J,(I: J) \in \mathcal{F}$, then $I \in \mathcal{F}$. This follows since $J$ being a multiplication ideal implies that $I=J \cdot(I: J)$, which is in $\mathcal{F}$ as $\mathcal{F}$ is monoidal. The second part of (1) follows from the first part, in view of Anderson's result $\left[\mathrm{An}_{2}:\right.$ p. 466] that (f.g.) multiplication ideals are closed under products. Finally, (2) clearly follows from (1).

Our next result shows that, as long as a ring $S$ is non-noetherian, a slightly bigger ring $R \supseteq S$ will have $\mathcal{F}_{\mathrm{fg}}(R)$ not strongly Oka.

Proposition 2.11. For any ring $S$, the following statements are equivalent:

(1) $S$ is a noetherian ring.

(2) $\mathcal{F}_{\mathrm{fg}}(R)$ is strongly Oka for every finitely generated $S$-algebra $R$.

(3) $\mathcal{F}_{\mathrm{fg}}(R)$ is strongly Oka for every module-finite $S$-algebra $R$.

Proof. $(1) \Rightarrow(2)$ is clear, since $S$ noetherian implies that the $S$-algebra $R$ in (2) is also noetherian, by the Hilbert Basis Theorem. $(2) \Rightarrow(3)$ is a tautology, so it is enough to prove $(3) \Rightarrow(1)$.

Assuming $S$ is non-noetherian, let us fix a non-f.g. ideal $K \triangleleft S$. Then $J:=$ $S \oplus S$ is a f.g. $S$-module with a non-f.g. submodule $I:=(0) \oplus K$. Furthermore, the quotient $S$-module $J / I \cong S \oplus(S / K)$ is faithful. Now consider the split-null ring extension $R=S \oplus J$ with the multiplication

$$
(s, j)\left(s^{\prime}, j^{\prime}\right)=\left(s s^{\prime}, s j^{\prime}+s^{\prime} j\right) \quad\left(\forall s, s^{\prime} \in S ; j, j^{\prime} \in J\right) .
$$

In this module-finite $S$-algebra, $I_{0}=(0, I) \subseteq J_{0}=(0, J)$ are ideals, with $J_{0}$ f.g., but $I_{0}$ not f.g., and $\left(I_{0}: J_{0}\right)$ consists of elements $(s, j)$ such that $(s, j)(0, J) \subseteq$ $(0, I)$. The last statement shows that, in $R,\left(I_{0}: J_{0}\right)=J_{0}\left(\operatorname{since}_{\operatorname{ann}_{S}}(J / I)=0\right)$,

\footnotetext{
${ }^{1}$ Borrowing a familiar catchy phrase: "to contain is to divide."
} 
so $\left(I_{0}: J_{0}\right)$ is also f.g., as is $\left(I_{0}, J_{0}\right)=J_{0}$. But $I_{0} \triangleleft R$ is not f.g., so $\mathcal{F}_{\text {fg }}(R)$ is not strongly Oka.

Let us now turn to the study of $\mathcal{F}_{\mathrm{pr}}(R)$ and its subfamilies. In [LR: (3.17)], we have shown that, for any ring $R$, any monoidal family $\mathcal{F} \subseteq \mathcal{F}_{\text {fg }}(R)$ is always strongly Oka. In view of the chart (2.7), it is natural to ask if the same family $\mathcal{F}$ satisfies $\left(\mathrm{P}_{3}\right)$. The answer is "no" in general, according to [LR: (3.18)]. However, we have the following result.

Proposition 2.13. For any family $\mathcal{F} \subseteq \mathcal{F}_{\mathrm{pr}}(R)$, the following are equivalent:

(1) $\mathcal{F}$ is an Ako family.

(2) $\mathcal{F}$ is a strongly Ako family.

(3) $\mathcal{F}$ has the property $\left(\mathrm{P}_{3}\right)$.

(4) $\mathcal{F}$ is closed under pairwise intersections, and whenever $(a) \in \mathcal{F}$ and $a \in K \triangleleft R, a K \in \mathcal{F}$.

Proof. By (2.7), we have (3) $\Rightarrow(2) \Rightarrow(1)$ (for general families). For the particular family $\mathcal{F}$ at hand, we further verify the following.

$(1) \Rightarrow(4)$. Assuming (1), consider any intersection $I=(a) \cap(b)$, where $(a),(b) \in$ $\mathcal{F}$. Since $(I, a)=(a) \in \mathcal{F}$ and $(I, b)=(b) \in \mathcal{F},(1)$ implies that $(I, a b) \in \mathcal{F}$. As $a b \in I$, this amounts to $I \in \mathcal{F}$. Next, suppose $a \in K \triangleleft R$, where $(a) \in \mathcal{F}$. Then $(a K, a)=(a) \in \mathcal{F}$ implies $\left(a K, a^{2}\right) \in \mathcal{F}$ (again by (1)). Since $a^{2} \in a K$, this amounts to $a K \in \mathcal{F}$.

$(4) \Rightarrow(3)$. Assuming (4), it suffices to check that $\mathcal{F}$ satisfies (Sq). Consider any ideal $I$ such that $(a)^{2} \subseteq I \subseteq(a)$, where $(a) \in \mathcal{F}$. Then $(I: a)$ contains $a$, so we have $I=a \cdot(I: a) \in \mathcal{F}$ by $(4)$.

Corollary 2.14. Let $\mathcal{F}=\mathcal{F}_{\mathrm{pr}}(R)$, where $R$ is an integral domain. Then each of the five properties for $\mathcal{F}$ on the top line of the chart (2.7) is equivalent to $R$ being $a$ PID.

Proof. If $\mathcal{F}$ is Ako (which is the weakest among the five properties), we claim that $R$ is a PID. Indeed, if $0 \neq K \triangleleft R$, then for any nonzero $a \in K,(2.13)(4)$ implies that $a K=(a k)$ for some $k \in K$, and hence $K=(k)$. Once we know $R$ is a PID, of course all five properties in question are valid for $\mathcal{F}$.

Without the domain assumption, we do not know exactly for what rings $R$ does $\mathcal{F}_{\text {pr }}(R)$ satisfy $\left(\mathrm{P}_{3}\right)$. In view of $(2.13)$ (for $\mathcal{F}=\mathcal{F}_{\text {pr }}(R)$ ), these are rather special rings: their principal ideals are closed under finite intersections, and every ideal in $R$ containing a non 0 -divisor must be principal (by an argument similar to that in the proof of (2.14)). Examples of such rings $R$ include all PIRs, and all von Neumann regular rings. [In the latter case, $\mathcal{F}_{\mathrm{pr}}(R)$ is monoidal and the property $(\mathrm{Sq})^{*}$ is tautological (as all ideals are idempotent), so $\mathcal{F}_{\mathrm{pr}}(R)$ satisfies even $\left(\mathrm{P}_{2}\right)$. For more general results on von Neumann regular rings, see $\S 4$.] Another example not of the above two types is given by a split-null extension $R=k \oplus M$ where $M$ is a vector space over a field $k$ (with $M^{2}=0$ ). Any proper principal ideal of $R$ has $k$-dimension $\leq 1$, and $a K=0$ whenever $a \in K \subseteq M$. These simple facts imply that $\mathcal{F}_{\text {pr }}(R)$ satisfies the condition (2.13)(4). 


\section{Semifilter Families and Doubleton Families}

In this section, we'll focus our attention on two other kinds of ideal families $\mathcal{F}$ in a ring $R$, and study the behavior of the various properties in (2.7) that can be imposed on $\mathcal{F}$. The two kinds of families are (1) semifilters, and (2) two-member families $\mathcal{F}=\{R, A\}$ (where $A \triangleleft R$ ). For these families, there are a few more relationships between the various properties, and for (2), all of these properties can be characterized directly via the ideal $A$ itself.

We'll start with the case of semifilters. In this case, it turns out that the first two vertical implications " $\left(\mathrm{P}_{3}\right) \Rightarrow$ Str. Oka" and "Str. Ako $\Rightarrow$ Oka" in (2.7) are reversible. (This means we are down to only five different properties in this chart.)

Proposition 3.1. Let $\mathcal{F}$ be a semifilter of ideals in $R$. Then

(1) $\mathcal{F}$ is Oka iff it is strongly Ako; and

(2) $\mathcal{F}$ is strongly Oka iff it satisfies $\left(\mathrm{P}_{3}\right)$.

Proof. The "if" parts of (1) and (2) are true in general, according to (2.7). Thus, it suffices to prove the "only if" parts, under the assumption that $\mathcal{F}$ is a semifilter. These have been proved before in [LR: (4.9), (4.12)] using categories of cyclic modules. Here, we'll prove them directly, without using categories.

For (2), we assume $\mathcal{F}$ is strongly Oka, and verify (2.4). Let $I, A, B \triangleleft R$ be such that $(I, A),(I, B) \in \mathcal{F}$; we need to show that $J:=(I, A B)$ is in $\mathcal{F}$. It is easy to see that $(J: A) \supseteq(I, B)$, so the semifilter assumption gives $(J: A) \in \mathcal{F}$. Since $(J, A)=(I, A B, A)=(I, A) \in \mathcal{F}$ too, the strongly Oka property gives $J \in \mathcal{F}$, as desired. Part (1) is proved similarly, by replacing $A$ by a principal ideal $(a)$.

If we are willing to impose a certain assumption on the complement family $\mathcal{F}^{\prime}=\{I \triangleleft R: I \notin \mathcal{F}\}$ of a semifilter $\mathcal{F}$, the following result shows somewhat surprisingly that all properties on $\mathcal{F}$ in the chart (2.7) are in fact equivalent to the Prime Ideal Principle!

Theorem 3.2. Let $\mathcal{F}$ be a semifilter of ideals in a ring $R$. Assume that every nonempty chain in $\mathcal{F}^{\prime}$ has an upper bound in $\mathcal{F}^{\prime}$ (with respect to inclusion). Then all properties in the following implication chart are equivalent:

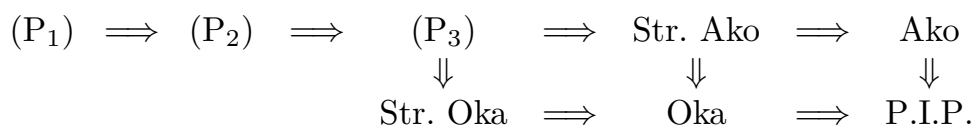

Here, by P.I.P., we mean the statement that all maximal members in $\mathcal{F}^{\prime}$ are prime ideals in $R$. In particular, the eight properties above are all equivalent for any semifilter $\mathcal{F}$ over a noetherian ring $R$.

Proof. We'll bypass (3.1), and show directly that, under the given assumptions, if $\mathcal{F}$ is not monoidal, then the Prime Ideal Principle cannot hold. This will prove the theorem, as "monoidal semifilter" is synonymous with "monoidal filter". Say $A, B \in \mathcal{F}$ are such that $A B \notin \mathcal{F}$. The hypothesis imposed on $\mathcal{F}^{\prime}$ enables us to apply Zorn's Lemma to get a maximal member $P$ of $\mathcal{F}^{\prime}$ with $A B \subseteq P$. Since $P \notin \mathcal{F}$, we have $A, B \nsubseteq P$ (by the semifilter property), so $P$ is not a prime ideal. This shows that P.I.P. fails. 
Even if Zorn's Lemma is not applicable to $\mathcal{F}^{\prime}$, a different argument can be used to prove a partial monoidal property for semifilters $\mathcal{F}$ that are Ako. In the case where $R$ is noetherian, the case $I=0$ of the following result gives another view for the equivalence of the seven properties in (2.7) (that is, without P.I.P.) for a semifilter $\mathcal{F}$.

Proposition 3.3. Let $\mathcal{F}$ be an Ako semifilter in a ring $R$. Then for any ideals $I, A, B \subseteq R$ with $A, B$ f.g., we have

$$
(I, A),(I, B) \in \mathcal{F} \Longrightarrow(I, A B) \in \mathcal{F}
$$

that is, the property (2.4) holds for all f.g. ideals $A, B \triangleleft R$.

Proof. Letting $A=\left(a_{1}, \ldots, a_{m}\right)$ and $B=\left(b_{1}, \ldots, b_{n}\right)$, we induct on $m+n$ $(\forall I \triangleleft R)$. The claim is trivial if $m=0$ or $n=0$, and is covered by the Ako property when $m=n=1$. Assuming that the claim holds for some value $m+n \geq 2$, we consider the case where $A=\left(a_{1}, \ldots, a_{m+1}\right)$ and $B=\left(b_{1}, \ldots, b_{n}\right)$. By symmetry, we may assume $m \geq 1$. Let $I \triangleleft R$ be such that $(I, A),(I, B) \in \mathcal{F}$. Defining $I_{0}=\left(I, a_{m+1}\right)$ and $A_{0}=\left(a_{1}, \ldots, a_{m}\right)$, we have $\left(I_{0}, A_{0}\right)=(I, A) \in \mathcal{F}$, and

$$
\left(I_{0}, B\right) \supseteq(I, B) \in \mathcal{F} \Longrightarrow\left(I_{0}, B\right) \in \mathcal{F}
$$

by the semifilter property. Invoking the inductive hypothesis, we get

$$
\left(I_{0}, A_{0} B\right)=\left(I, A_{0} B, a_{m+1}\right) \in \mathcal{F} .
$$

Now, letting $I_{1}=\left(I, A_{0} B\right)$, we have $\left(I_{1}, a_{m+1}\right) \in \mathcal{F}$ and

$$
\left(I_{1}, B\right) \supseteq(I, B) \in \mathcal{F} \Longrightarrow\left(I_{1}, B\right) \in \mathcal{F} .
$$

Using the inductive hypothesis again, we have

$$
(I, A B)=\left(I, A_{0} B, a_{m+1} B\right)=\left(I_{1}, a_{m+1} B\right) \in \mathcal{F} .
$$

Note that the ideal $B$ has remained unchanged throughout the above induction. Thus, the same inductive argument will also show the following: if $\mathcal{F}$ is a strongly Ako semifilter, then the property (2.4) holds for all $B \triangleleft R$ and all f.g. $A \triangleleft R$.

In the second part of this section, we'll study the case of a doubleton family $\mathcal{F}=\{R, A\}$, where $A$ is a fixed proper ideal of $R$. The possibility of exploring this peculiar case eluded our attention in $[\mathrm{LR}]$, but this case turns out to be, in some sense, diametrically opposite to the case of semifilters. What does it mean (in terms of $A$ ) for such a family $\mathcal{F}=\{R, A\}$ to be Oka, Ako, monoidal, ..., etc.? Quite pleasantly, the attempt to answer this question brought our study into direct contact with various basic issues in commutative algebra. As it turned out, in working with a two-member family $\mathcal{F}=\{R, A\}$, an essential object to consider is the $R / A$-module $A / A^{2}$. In the standard literature, this is called the conormal module of the ideal $A$; see, e.g. [Ku: p. 149]. The two most important cases are those in which we want $\mathcal{F}$ to be Oka or strongly Oka. These cases will be worked out in detail in the next two results.

Theorem 3.4. For $\mathcal{F}=\{R, A\}$ where $A \neq R$, the following are equivalent:

(1) $\mathcal{F}$ is strongly Oka.

(2) $\left(A^{2}: A\right) \neq A$; that is, the conormal $R / A$-module $A / A^{2}$ is not faithful. 
(3) There is no ideal $I \triangleleft R$ such that $A=(I: A)$.

(4) There are no ideals $I, J \triangleleft R$ such that $A=(I, J)=(I: J)$.

If (1) holds, we'll say $A$ is a strongly Oka ideal.

Proof. $(1) \Rightarrow(2)$. Assume $\left(A^{2}: A\right)=A$. This together with $\left(A^{2}, A\right)=A \in \mathcal{F}$ implies that $A^{2} \in \mathcal{F}$, by (1). Since $A^{2} \subseteq A \subsetneq R$, we have $A^{2}=A$. Now $A=$ $\left(A^{2}: A\right)=(A: A)=R$, a contradiction.

$(2) \Rightarrow(3)$. Suppose $(I: A)=A$ for some $I \triangleleft R$. Then $A^{2} \subseteq I \subseteq A$, and $A / I$ is a faithful $R / A$-module. Since we have a natural epimorphism $A / A^{2} \rightarrow A / I, A / A^{2}$ is also a faithful $R / A$-module.

$(3) \Rightarrow(4)$. If $I, J \triangleleft R$ are such that $A=(I, J)=(I: J)$, then $(I: A)=$ $(I:(I, J))=(I: J)=A$, contradicting (3).

$(4) \Rightarrow(1)$. Assuming (4), we must check $(I, J),(I: J) \in \mathcal{F} \Rightarrow I \in \mathcal{F}$. By (4), $(I, J),(I: J)$ cannot both be $A$, so one of them is $R$. If $(I, J)=R$, then

$$
I=(I: R)=(I:(I, J))=(I: J) \in \mathcal{F} .
$$

If $(I: J)=R$, then $1 \in(I: J) \Rightarrow J \subseteq I$, so $I=(I, J) \in \mathcal{F}$ as well.

To handle the Oka case, we recall a useful definition from the theory of modules (see, e.g. [La 3 : p. 32]). An element $\alpha$ in an $S$-module $M$ (over any ring $S$ ) is said to be unimodular if $f(\alpha)=1$ for some $f \in \operatorname{Hom}_{S}(M, S)$; or equivalently, $S \cdot \alpha$ is free on the basis $\{\alpha\}$ and is a direct summand of $M$. Thus, $M$ has a unimodular element iff it has a quotient module isomorphic to $S$. A necessary (though not sufficient) condition for this is that $M$ is a faithful $S$-module.

Theorem 3.5. For $\mathcal{F}=\{R, A\}$ where $A \neq R$, the following are equivalent:

(1) $\mathcal{F}$ is Oka.

(2) The conormal $R / A$-module $A / A^{2}$ has no unimodular element.

(3) There is no ideal $I \subseteq A$ with $A / I$ cyclic such that $A=(I: A)$.

(4) We cannot have $A=(I, a)=(I: a)$ where $I \triangleleft R$ and $a \in R$.

If (1) holds, we'll say $A$ is an Oka ideal.

Proof. For $(3) \Rightarrow(4)$ and $(4) \Rightarrow(1)$, we can repeat the proofs of the same implications in Thm. 3.4 above, with $J$ replaced by a principal ideal $(a)$.

(1) $\Rightarrow(2)$. Assuming (2) fails, there exists an ideal $I$ between $A^{2}$ and $A$ such that $A / I \cong R / A$ (as $R / A$-modules). Then $A / I$ is cyclic, so $A=(I, a)$ for some $a \in A$. The isomorphism $A / I \cong R / A$ then shows that $A=(I: a)$. Since $(I, a),(I: a) \in \mathcal{F}$ and $\mathcal{F}$ is Oka, we have $I \in \mathcal{F}$, and so $I=A$. But now $A=(I: a)=(A: a)=R$, a contradiction.

$(2) \Rightarrow(3)$. Suppose $I \subseteq A$ exists with $A / I$ cyclic and $(I: A)=A$. Write $A=(I, a)$. Then $A=(I:(I, a))=(I: a)$. Now $A^{2} \subseteq I$, and multiplication by $a$ induces an isomorphism $R / A \cong A / I$. Thus, $A / A^{2}$ has a quotient module $A / I \cong R / A$, contradicting (2).

As for the remaining cases ( $\frac{1}{2}$-monoidal, Ako, strongly Ako, and the three properties $\left.\left(\mathrm{P}_{i}\right)\right)$, we have the following parallel result. 
Theorem 3.6. For any ring $R$, let $\mathcal{F}=\{R, A\}$, where $A \neq R$ is an ideal.

(1) $\mathcal{F}$ satisfies $\left(\mathrm{P}_{1}\right)$ iff $A^{2}=A$ and $A$ is a maximal ideal.

(2) $\mathcal{F}$ satisfies $\left(\mathrm{P}_{2}\right)$ iff, for any $C \triangleleft R, C^{2}=A \Leftrightarrow C=A$.

(3) $\mathcal{F}$ satisfies $\left(\mathrm{P}_{3}\right)$ iff $\mathcal{F}$ is monoidal, iff $A^{2}=A$.

(4) $\mathcal{F}$ is $\frac{1}{2}$-monoidal iff $A$ is not principal or $A=(e)$ where $e^{2}=e$.

(5) $\mathcal{F}$ is Ako iff $\mathcal{F}$ is strongly Ako; iff the conormal module $A / A^{2}$ has no simple quotient; iff we cannot have $A=(I, a)$ where $I \triangleleft R$ and $a^{2} \in I \subsetneq A$. In this case, we say $A$ is an Ako ideal.

(6) Idempotent ideals are Ako, and Ako (and strongly Oka) ideals are Oka.

Proof. (1)-(4) are easily checked from definitions, and (5) can be shown along the same lines as in the proofs of (3.4) and (3.5) above. ${ }^{2}$ To avoid repetitions, we omit the details. Part (6) follows from (3), (5) and the chart (2.7) (invoked for two-member families). Of course, it is also easy to verify (6) directly from the ideal-theoretic conditions on $A$ given in (5) above, and in (3.4), (3.5).

In a number of cases, the converse of the first part of (6) is also true, as the following result shows.

Proposition 3.7. An Ako ideal $A \triangleleft R$ must be idempotent in the following cases: (1) $A$ is f.g.; and (2) R/A is a perfect ring in the sense of Bass [Ba].

Proof. (1) Assume $A$ is f.g. If $A^{2} \neq A$, the nonzero f.g. module $A / A^{2}$ has a maximal submodule, and therefore has a simple quotient. This contradicts one of the conditions in $(3.6)(5)$. Thus, we must have $A^{2}=A$. In fact, in this case, a standard result in commutative algebra (see, e.g. [La $:(2.43)]$ ) implies that $A=e R$ for some $e=e^{2} \in R$. (This fact was also needed for the proof of (3.6)(4) above.)

(2) If $R / A$ is a perfect ring, a well-known result of Bass [Ba: p. 470] (see also [La 2 : Ex. 24.7]) guarantees that every nonzero $R / A$-module has a maximal submodule. Case (2) follows by applying this to the conormal module $A / A^{2}$. (In fact, considerably weaker conditions on the commutative ring $R / A$ would have sufficed, by a result of Hamsher and Renault: see [La2: Ex. 24.9].)

Corollary 3.8. (Principal Ideal Case) Let $A=(r) \neq R$. Then

(1) $A$ is Ako iff $r$ is von Neumann regular; that is, $r=r x r$ for some $x \in R$;

(2) $A$ is Oka iff $A$ is strongly Oka, iff $\operatorname{ann}(r) \not \subset(r)$;

(3) if $R$ is a domain, any of the above conditions holds iff $r=0$.

Proof. (1) follows from (3.6)(5) and (3.7)(1). The equivalence of "Oka" and "Strongly Oka" in (2) follows from the fact that the cyclicity condition (on $A / I$ ) in $(3.5)(3)$ is free. For the last part of $(2)$, note that $\left((r)^{2}: r\right)=(r$, ann $(r))$ (for any $r \in R)$. From this, it is clear that $\left((r)^{2}: r\right) \neq(r)$ iff ann $(r) \nsubseteq(r)$. Finally, (3) is clear from (1) and (2).

One somewhat surprising consequence of Theorems (3.4)-(3.6) is the following proposition, which to some extent illustrates the role of two-member families in the consideration of ideal families in general.

\footnotetext{
${ }^{2}$ One small additional step needed for the proof of part (5) is that the word "simple" there can also be replaced by "nonzero cyclic".
} 
Proposition 3.9. If $\mathcal{F}$ is any non-singleton Oka (resp. Ako, strongly Oka) family of ideals in $R$, then any minimal member $A \in \mathcal{F}$ is Oka (resp. Ako, strongly Oka).

Proof. Suppose $A=(I, a)=(I: a)$, where $a \in R$ and $I \triangleleft R$. Then $(I, a)$, $(I: a) \in \mathcal{F}$, so $\mathcal{F}$ being Oka implies that $I \in \mathcal{F}$. But then $I=A$ by the minimality of $A$, and we end up with $A=(I: a)=(A: a)=R$, a contradiction. Similar arguments work in the case of Ako and strongly Oka families.

To place this result in perspective, we can make the following two "parallel" statements, where the first one is just the Prime Ideal Principle (1.3) dressed up in an "iff" form - taking into account an earlier remark we've made after the proof of [LR: (2.12)].

- An ideal is prime iff it is maximal w.r.t. not being in a certain Oka family;

- A proper ideal is Oka iff it is minimal w.r.t. being in a certain Oka family.

Of course, these statements also hold with the word "Oka" replaced by "Ako" or "Strongly Oka".

Coming back to the case $|\mathcal{F}|=2$, with the explicit interpretation of the various properties on $\mathcal{F}=\{R, A\}$ in (3.4)-(3.6), we can now prove the following result.

Theorem 3.10. For $\mathcal{F}=\{R, A\}$ where $A \neq R$, the implication "Str. Ako $\Rightarrow$ Ako" in the chart (2.7) is reversible, but each of the six other implications is irreversible.

Proof. The first conclusion is just a part of (3.6)(5). (In view of this, we may safely confuse "Ako" with "Strongly Ako" for the rest of $\S 3$.

$\left(\mathrm{P}_{2}\right) \nRightarrow\left(\mathrm{P}_{1}\right)$ : this is shown by taking $R=\mathbb{Z}_{6}$ and $A=(0)$.

$\left(\mathrm{P}_{3}\right) \nRightarrow\left(\mathrm{P}_{2}\right)$ : this is shown by taking $R=\mathbb{Z}_{12}$ and $A=(4)$, noting that $(4)=(4)^{2}=(2)^{2}$.

Next, we'll show that neither one of "strongly Oka" and "strongly Ako" could imply the other (for two-member families). Once this is shown, it follows right away from (2.6) that each of the four "legs" of the square in the chart (2.7) is an irreversible implication (for two-member families). This will complete the proof of the theorem at hand.

"Str. Oka $\nRightarrow$ Str. Ako": It suffices to produce a nonzero strongly Oka ideal $A$ in some noetherian domain $R$, for then $A^{2} \subsetneq A$ by [La: (2.43)] as before, so $A$ is not Ako by (3.7)(1). Following a suggestion of Vijay Kodiyalam, we work in the polynomial ring $R=\mathbb{Q}[x, y]$, and take $A$ to be the ideal generated by all monomials of degree $2 k$ except $x^{k} y^{k}$. If $k>1$, we see easily that $A^{2}=(x, y)^{4 k}$. Thus, $x^{k} y^{k} \cdot A \subseteq(x, y)^{4 k}=A^{2}$. But $x^{k} y^{k} \notin A$, showing that $\left(A^{2}: A\right) \supsetneq A$. (More precisely, $\left(A^{2}: A\right)=(x, y)^{2 k}$.)

By working a little harder, we can also produce an example where $A$ is a binary generated ideal. For instance, take the noetherian $\operatorname{ring} R=\mathbb{Q}[x, y, z]$ with the relations

$$
z x=x^{2}+y^{2}, z y=x^{2}-y^{2}, \text { and } z^{2}=(x-y) z+2 x y .
$$

According to Singular, $R$ is an integral domain of Krull dimension 1. For the nonzero ideal $A=(x, y) \triangleleft R$, the first two relations in (3.11) imply that $z A \subseteq A^{2}$, 
and it is easy to check (upon lifting to the polynomial ring $\mathbb{Q}[X, Y, Z]$ ) that $z \notin A$. Thus, we have again $\left(A^{2}: A\right) \supsetneq A$, so $A$ is strongly Oka, as desired.

"Str. Ako $\nRightarrow$ Str. Oka": For this, we have to work in the non-noetherian case (since otherwise $(3.7)(1)$ gives "Str. Ako $\Leftrightarrow\left(\mathrm{P}_{3}\right) \Rightarrow$ Str. Oka" for $\left.|\mathcal{F}|=2\right)$. Such an example can be constructed by using a 1-dimensional non-noetherian valuation domain. Let $K$ be any field with a Krull valuation $v: K \rightarrow \mathbb{Q} \cup\{\infty\}$, where $\mathbb{Q}$ is viewed as an ordered abelian group under its usual ordering. (For the existence of such a field $K$, see [Ri:p.368].) Let $R \subseteq K$ be the corresponding valuation domain, and let

$$
A:=\{r \in R: v(r)>\sqrt{2}\} \triangleleft R .
$$

Since $v(A)$ does not have a smallest element, $A$ is not a principal ideal. If $I$ is any ideal properly contained in $A$, then $A / I$ cannot be cyclic. (Otherwise, $A=(I, a)$ for some $a \in A \backslash I$, but $(a) \nsubseteq I \Rightarrow I \subseteq(a) \Rightarrow A=(a)$.) Therefore, by (3.6)(5), the ideal $A$ is Ako. We finish by checking that $A \subseteq\left(A^{2}: A\right)$ is an equality (for then $\mathcal{F}$ is not strongly Oka by (3.4)). By an easy computation,

$$
A^{2}:=\{r \in R: v(r)>2 \sqrt{2}\} .
$$

Thus, for any $r \in\left(A^{2}: A\right)$, we have $v(r)+v(A)>2 \sqrt{2}$. This implies that $v(r)>\sqrt{2}$, so $r \in A$, as desired. [Note that the choice of the value group is important for this example! If we had chosen the value group to be $\mathbb{R}$ (again with the usual ordering), the construction in (3.12) would have produced an ideal $A$ such that $A \subsetneq\left(A^{2}: A\right)$, since an element $x \in R$ with $v(x)=\sqrt{2}$ would be in $\left(A^{2}: A\right) \backslash A$. In this case, $A$ would be both Ako and strongly Oka.]

To complete this section, we collect a few types of examples of ideals $A$ for which $\left(A^{2}: A\right)=A$. By (3.4), these ideals are not strongly Oka. The first example $((3.16)(1)$ below) is made possible by the following well-known observation (which will also be needed in $\S 5$ below).

Proposition 3.14. For any ring $R$, let $A, B \triangleleft R$ be such that $A$ is f.g., with ann $(A) \subseteq B$. Then $(A B: A) \subseteq \sqrt{B}$.

Proof. By working with a set of $n$ generators for $A$, the usual "determinant trick" in commutative algebra shows that every $r \in(A B: A)$ satisfies an equation

$$
\left(r^{n}+b_{1} r^{n-1}+\cdots+b_{n}\right) \cdot A=0
$$

for some $b_{i} \in B$ and $n \geq 1$. Thus $r^{n} \in-\left(b_{1} r^{n-1}+\cdots+b_{n}\right)+\operatorname{ann}(A) \subseteq B$.

Examples 3.16. (1) Let $A \triangleleft R$ be a f.g. faithful radical ideal. Then (3.14) (applied with $B=A)$ shows that $\left(A^{2}: A\right)=A$.

(2) (Non-radical examples). Let $J \triangleleft R$ be such that the associated graded ring $\operatorname{Gr}_{J}(R)=\bigoplus_{i \geqslant 0} J^{i} / J^{i+1}$ is a domain. Then $A=J^{n}$ satisfies $\left(A^{2}: A\right)=A$ (for all $n \geq 1)$. To see this, assume there exists $r \in\left(A^{2}: A\right) \backslash A$. Let $r \in J^{i}$ with $i<n$ chosen largest. Then $r+J^{i+1} \neq 0 \in J^{i} / J^{i+1}$, and $n \geq i+1$. For every $a \in A$, we have

$$
\left(r+J^{i+1}\right)\left(a+J^{n+1}\right)=r a+J^{n+i+1}=0 \in \operatorname{Gr}_{J}(R),
$$


since $r a \in J^{2 n}$ and $2 n \geq n+i+1$. This contradicts the fact that $\operatorname{Gr}_{J}(R)$ is a domain.

(3) Let $A \triangleleft R$ be such that, as an $R / A$-module, $A / A^{2}$ contains a free module of rank 1 . Then $A / A^{2}$ is surely faithful over $R / A$, and hence $\left(A^{2}: A\right)=A$.

(4) If $A \triangleleft R$ is an invertible ideal, then $r A \subseteq A^{2} \Rightarrow r \in A$ (upon multiplication by $\left.A^{-1}\right)$. Thus, $\left(A^{2}: A\right)=A$. (Of course, this works more generally for all cancellation ideals $A \triangleleft R$ : see [Gi: II.5.4].)

As for non-Oka ideals, the following offers perhaps the easiest and most basic type of examples.

Proposition 3.18. A maximal ideal $A \triangleleft R$ with $A^{2} \neq A$ is non-Oka. (In particular, if $R$ is a local ring with a f.g. maximal ideal $A \neq 0$, then $A$ is not Oka.)

Proof. Since $R / A$ is a field, any nonzero vector in the $R / A$-vector space $A / A^{2}$ is unimodular. The last statement follows easily from Nakayama's Lemma.

Much more can be said about Oka/non-Oka ideals and the difference between Oka and strongly Oka ideals. In fact, the new connections found above between the conormal module $A / A^{2}$ and the notion of Oka ideals etc. have led to some interesting new results on the minimal number of generators for certain ideals. These will be reported later in $[\mathrm{KLR}]$.

\section{Von Neumann Regular Rings}

In this section, we turn our attention to von Neumann regular rings; that is, rings $R$ in which all elements are von Neumann regular. For such $R$, all ideals are idempotent, so they are automatically Ako and strongly Oka. But this only means that we should look beyond two-member ideal families. For von Neumann regular rings, the various ideal family properties have certain special relationships and characterizations that are not valid for other classes of rings. We shall record (and prove) some of these below. To begin with, there is a nice simplification of the properties $\left(\mathrm{P}_{2}\right)$ and $\left(\mathrm{P}_{3}\right)$ on the left part of the chart $(2.7)$, as follows.

Proposition 4.1. For an ideal family $\mathcal{F}$ in a von Neumann regular ring $R$ with $R \in \mathcal{F},\left(\mathrm{P}_{2}\right)$ and $\left(\mathrm{P}_{3}\right)$ are each equivalent to $\mathcal{F}$ being monoidal. Under the latter condition, $\mathcal{F}$ is strongly Oka and strongly Ako. If an ideal $M \triangleleft R$ is maximal with respect to $M \notin \mathcal{F}$, then $M$ is a maximal ideal of $R$.

Proof. Since all ideals are idempotent, the squeezing properties $(\mathrm{Sq})$ and $(\mathrm{Sq})^{*}$ are automatic. In fact, we have even $I J=I \cap J$ for all $I, J \triangleleft R$, so $\left(\mathrm{P}_{2}\right)$ and $\left(\mathrm{P}_{3}\right)$ both boil down to $\mathcal{F}$ being monoidal. Under the latter condition, (2.7) implies that $\mathcal{F}$ is strongly Oka and strongly Ako. If $M \triangleleft R$ is maximal w.r.t. $M \notin \mathcal{F}$, P.I.P. implies that $M$ is a prime ideal in $R$. Since $R$ has Krull dimension 0, this means that $M$ is a maximal ideal in $R$.

Proposition 4.1 enables us to name a large number of examples of strongly Oka and strongly Ako ideal families in a von Neumann regular ring $R$. For instance, any monoidal family of principal ideals has these properties (as is also clear from 
(2.13)). If we fix any ideals $A, B \triangleleft R$, the family of ideals containing $A$ satisfies all properties in (2.7), and each of the families

$$
\{R, A\}, \quad\{I \triangleleft R: I=R \text { or } I \subseteq A\}, \quad \text { and } \quad\{R, A, B, A \cap B\}
$$

satisfies all properties in (2.7) with the obvious exception of $\left(\mathrm{P}_{1}\right)$.

In the case of two-member families, (3.6)(6) says that "Ako $\Rightarrow$ Oka" over any $R$. It turns out that the same implication holds for all families over any von Neumann regular ring. More generally, (1) of the following proposition offers a useful sufficient condition for this to happen in a given ring $R$.

Proposition 4.3. Let $R$ be any ring.

(1) If, for every $a \in R$ and $I \triangleleft R,(I: a)=(I, b)$ for some $b \in R$, then every Ako family $\mathcal{F}$ in $R$ is Oka.

(2) If $I \triangleleft R$ and $e=e^{2} \in R$, then $(I: e)=(I, 1-e)$.

(3) In a von Neumann regular ring $R$, any Ako family $\mathcal{F}$ is Oka.

Proof. (1) Suppose $(I, a),(I: a) \in \mathcal{F}$. To show that $I \in \mathcal{F}$, write $(I: a)=(I, b)$ as in the hypothesis of $(1)$. Since $\mathcal{F}$ is Ako, we have $(I, a b) \in \mathcal{F}$. But $a b \in I$, so this amounts to $I \in \mathcal{F}$.

(2) " $\supseteq$ " is clear, and " $\subseteq$ " follows from the equation $r=r e+r(1-e)$.

(3) If $R$ is von Neumann regular, every principal ideal $(a) \triangleleft R$ has the form $(e)$ for some $e=e^{2} \in R$. Then, for any $I \triangleleft R,(I: a)=(I: e)=(I, 1-e)$ by (2), so the hypothesis in (1) is satisfied. Thus, (3) follows from (1).

In a general von Neumann regular ring, Oka and Ako families need not be monoidal. However, they turn out to be characterizable by certain partial monoidal properties, as we will show in (4.4) and (4.9) below.

Theorem 4.4. Let $\mathcal{F}$ be an ideal family in a von Neumann regular ring $R$ with $R \in \mathcal{F}$. The following are equivalent:

(1) $\mathcal{F}$ is Oka.

(2) For $e=e^{2} \in R$ and $I \triangleleft R,(I, e),(I, 1-e) \in \mathcal{F} \Rightarrow I \in \mathcal{F}$.

(3) $\mathcal{F}$ is "comaximally monoidal", in the sense that

$$
A, B \in \mathcal{F} \text { and } A+B=R \Longrightarrow A B \in \mathcal{F} \text {. }
$$

Proof. $(1) \Leftrightarrow(2)$ is clear from the proof of (4.3)(3).

$(3) \Rightarrow(2)$. Suppose $(I, e),(I, 1-e) \in \mathcal{F}$, as in (2). Since these two ideals are comaximal in $R,(3)$ implies that their product is in $\mathcal{F}$. But this product is $\left(I^{2}, I e, I(1-e)\right)=(I, I e, I(1-e))=I$, so $I \in \mathcal{F}$.

$(2) \Rightarrow(3)$. We shall prove this implication for any exchange ring $R$. (In particular, it will hold for any von Neumann regular ring; see [Wa].) Let $A, B \in \mathcal{F}$ be comaximal. By the exchange property (as elucidated in [Ni]), there exists an idempotent $e \in R$ such that $e \in A$ and $1-e \in B$. For $I:=A B, A \supseteq(I, e)$ is an equality, since $a \in A$ implies that

$$
a=a(1-e)+a e \in A B+(e)=(I, e) .
$$

Similarly, we have $B=(I, 1-e)$. As $A, B \in \mathcal{F},(2)$ gives $A B=I \in \mathcal{F}$. 
Remark 4.6. (a) Note that the comaximally monoidal condition (4.5) holds automatically if $R$ is a local ring, since in that case $A+B=R \Rightarrow A=R$ or $B=R$ so $A B$ is just one of $A, B$. If $R$ is, in addition, von Neumann regular, (4.4) would say that any family $\mathcal{F}$ containing $R$ is Oka. This is not a contradiction, since in this case $R$ must be a field, and $\mathcal{F}$ can only be $\{R\}$ or $\{R,(0)\}$, both of which are monoidal filters.

(b) From the characterization (4.4)(2) alone, it is once again clear that "Ako $\Rightarrow$ Oka" in von Neumann regular rings. On the other hand, the characterization (4.4)(3) enables us to produce many Oka families in such rings. For instance, if $\left\{A_{i}\right\}$ is any family of ideals that are pairwise not comaximal, then $\left\{A_{i}\right\}$ together with the unit ideal $R$ provide an Oka family $\mathcal{F}$ in $R$, as is immediately verified through (4.4)(3).

Example 4.7. Using the idea just mentioned in (4.6)(b) above, we can construct a strongly Oka family $\mathcal{F}$ in a von Neumann regular ring that is not $\frac{1}{2}$-monoidal, and therefore not Ako. (Note that, in any ring, Ako families are always $\frac{1}{2}$-monoidal, by taking $I=0$ in Def. (1.2).) Indeed, let $R=A \times B \times C$ where $A, B, C$ are fields, and let $e_{1}, e_{2}$ be the identities of $A$ and $B$ (viewed as elements of $R$ ). The family $\mathcal{F}=\{R, A, B\}$ is Oka by (4.6)(b) (and hence strongly Oka since $R$ is a PIR). We have $\left(e_{1}\right)=A \in \mathcal{F}$ and $\left(e_{2}\right)=B \in \mathcal{F}$, but $\left(e_{1}\right)\left(e_{2}\right)=(0) \notin \mathcal{F}$, so $\mathcal{F}$ is not $\frac{1}{2}$-monoidal.

Example 4.8. A suitable modification of the construction method in (4.7) can be used to show that, in a von Neumann regular ring $R$, an Oka family need not be strongly Oka. For this, of course we have to work with a non-noetherian $R$. Let $R=A \times B \times C \times D \times \cdots$ be an infinite direct product of fields, and let

$$
\mathcal{F}=\{R, A \oplus B, B \oplus C \oplus D \oplus \cdots\} .
$$

This is an Oka family by (4.6)(b). Let $P=C \oplus D \oplus \cdots$. Then $(B, P) \in \mathcal{F}$, and a quick calculation shows that $(B: P)=A \oplus B \in \mathcal{F}$. However, $B \notin \mathcal{F}$, so $\mathcal{F}$ is not strongly Oka.

In parallel to (4.4), we record also some characterizations of Ako families in von Neumann regular rings in terms of their idempotents.

Theorem 4.9. Let $\mathcal{F}$ be an ideal family in a von Neumann regular ring $R$ with $R \in \mathcal{F}$. The following are equivalent:

(1) $\mathcal{F}$ is Ako.

(2) For idempotents $e, e^{\prime} \in R$ and $I \triangleleft R,(I, e),\left(I, e^{\prime}\right) \in \mathcal{F} \Rightarrow\left(I, e e^{\prime}\right) \in \mathcal{F}$.

(3) For orthogonal idempotents $e, e^{\prime} \in R$ and $I \triangleleft R,(I, e),\left(I, e^{\prime}\right) \in \mathcal{F} \Rightarrow I \in \mathcal{F}$.

(4) If $A, B \triangleleft R$ are such that $(A+B) / A B$ is cyclic, then $A, B \in \mathcal{F} \Rightarrow A B \in \mathcal{F}$.

Proof. $(1) \Leftrightarrow(2)$ follows again from the fact that, in a von Neumann regular ring, any principal ideal is generated by an idempotent.

$(1) \Rightarrow(4)$. Let $A, B$ be as in (4). Since $R$ is von Neumann regular, $A B=A \cap B$, so in $\bar{R}=R / A B, \bar{A} \cap \bar{B}=\{0\}$. But by assumption $\bar{A} \oplus \bar{B}$ is cyclic. Thus, $\bar{A}, \bar{B}$ are cyclic (each being a quotient of $\bar{A} \oplus \bar{B})$. Say $\bar{A}=(\bar{a})$ and $\bar{B}=(\bar{b})$. Then $A=(A B, a)$, and $B=(A B, b)$. By the Ako property, we have $(A B, a b) \in \mathcal{F}$. Since $a b \in A B$, this simplifies to $A B \in \mathcal{F}$. 
$(4) \Rightarrow(3)$. For $I, e, e^{\prime}$ be as in $(3)$, let $A=(I, e)$ and $B=\left(I, e^{\prime}\right)$. Then $A, B \in \mathcal{F}$, and $A B=I$. Now

$$
(A+B) / A B=\left(I, e, e^{\prime}\right) / A B=\left(I, e+e^{\prime}\right) / I
$$

is cyclic. Thus, $(4) \Rightarrow A B \in \mathcal{F}$; that is, $I \in \mathcal{F}$.

$(3) \Rightarrow(2)$. Let $e, e^{\prime} \in R$ be idempotents with $(I, e),\left(I, e^{\prime}\right) \in \mathcal{F}$, where $I \triangleleft R$.

Let $J=\left(I, e e^{\prime}\right)$, and write $f=1-e, f^{\prime}=1-e^{\prime}$. Then

$$
\left(J, e f^{\prime}\right)=\left(I, e e^{\prime}, e f^{\prime}\right)=(I, e) \in \mathcal{F},
$$

and similarly $\left(J, f e^{\prime}\right)=\left(I, e^{\prime}\right) \in \mathcal{F}$. Since $e f^{\prime}$ and $f e^{\prime}$ are idempotents, (3) implies that $\left(I, e e^{\prime}\right)=J \in \mathcal{F}$.

Since Ako families are Oka in a von Neumann regular ring $R$ (according to (4.3)(3)), it might seem tempting to speculate that strongly Ako families are also strongly Oka in $R$. This is, of course, true in the noetherian case, as $R$ must be a PIR under the noetherian assumption. However, the implication does not hold in the non-noetherian case, as the following example shows.

Example 4.10. Let $R$ be a von Neumann regular ring with a nonprincipal ideal $J$ whose annihilator $K=\operatorname{ann}(J)$ is also nonprincipal. (The ring $R$ consisting of sequences in $\mathbb{Q} \times \mathbb{Q} \times \cdots$ that are eventually constant will work: we can take

$$
J=R e_{1} \oplus R e_{3} \oplus \cdots \triangleleft R, \quad \text { with annihilator } K=R e_{2} \oplus R e_{4} \oplus \cdots \triangleleft R,
$$

where $e_{i}$ are the "unit-vector" idempotents. It is easy to check that neither $J$ nor $K$ is principal in $R$.) Consider the family $\mathcal{F}=\{R, J, K\}$, which is not strongly Oka since $(0, J)=J \in \mathcal{F}$ and $(0: J)=K \in \mathcal{F}$, but $(0) \notin \mathcal{F}$. We claim that $\mathcal{F}$ is strongly Ako. To see this, suppose $(I, a),(I, B) \in \mathcal{F}$, where $I, B \triangleleft R$ and $a \in R$. Since $I^{2}=I$, we have as usual

$$
(I, a B)=(I, a)(I, B)=(I, a) \cap(I, B),
$$

and we need to check that $(I, a B) \in \mathcal{F}$. This is clear if either $(I, a)=R$, or $(I, B)=R$, or $(I, a)=(I, B)$. Thus, the only case not yet covered is where

$$
(I, a)=J,(I, B)=K, \quad \text { or }(I, a)=K,(I, B)=J .
$$

In either case, (4.12) shows that $I \subseteq J K=0$. But then we have $J=(a)$ or $K=(a)$, a contradiction. (Note that $\mathcal{F}$ also fails to be monoidal, since it contains $J, K$, but not $J K=0$.)

\section{Partial Monoidal Properties and Integral Domains}

Although Oka and strongly Oka families need not be monoidal in general (by the examples in $\S \S 3-4)$, they do have certain partial closure properties under the multiplication of ideals. In the case of von Neumann regular rings, for instance, we saw in $\S 4$ that "Oka" is equivalent to the "comaximally monoidal" property defined in (4.5). While this may not be indicative of what happens in general, other considerations can be used to identify some closure properties of Oka (and strongly Oka) families under multiplication, as we'll show in this section. 
Lemma 5.1. Let $\mathcal{F}$ be an Oka family in a ring $R$, and let $a \in R$ and $B \triangleleft R$ be such that ann $(a) \subseteq B$. If $(a), B \in \mathcal{F}$, then $a B \in \mathcal{F}$. In particular, this implication holds if $a$ is a non 0-divisor or if $(a, B)=R$.

Proof. Assume that $(a), B \in \mathcal{F}$. Note that $(a B: a) \supseteq B$ is an equality. Indeed, if $r \in(a B: a)$, then $a r=a b$ for some $b \in B$, so $r \in b+\operatorname{ann}(a) \subseteq B$. Therefore, $(a B: a)=B \in \mathcal{F}$. Since $(a B, a)=(a) \in \mathcal{F}$ also, the Oka property gives $a B \in \mathcal{F}$.

If $a$ is a non 0-divisor, $\operatorname{ann}(a)=(0) \subseteq B$. On the other hand, if $(a, B)=R$, fix an equation $a x+b^{\prime}=1$, where $x \in R$ and $b^{\prime} \in B$. For any $y \in$ ann $(a)$, we have $y=y\left(a x+b^{\prime}\right)=y b^{\prime} \in B$, so again ann $(a) \subseteq B$. In either case, the main conclusion of the Lemma applies.

Proposition 5.2. An Oka family $\mathcal{F}$ in a domain $R$ is always $\frac{1}{2}$-monoidal.

Proof. Let $(a) \in \mathcal{F}, B \in \mathcal{F}$. If $a=0$, we have of course $a B=(0)=(a) \in \mathcal{F}$. If $a \neq 0$, then $a$ is a non 0 -divisor in $R$, and (5.1) gives $a B \in \mathcal{F}$.

Remark 5.3. Recall from Example 4.7 that the conclusion in (5.2) is not true (even for strongly Oka families) if $R$ is not assumed to be a domain.

We do not know precisely how much a difference exists between an Oka family and a $\frac{1}{2}$-monoidal family in a domain $R$. A relevant point regarding this question is that, while it is not clear how to "determine" all Oka families in $R$, the following result does give the construction for all $\frac{1}{2}$-monoidal families in a domain $R$.

Theorem 5.4. Let $\mathcal{H}$ be any monoidal family of principal ideals in an integral domain $R$, and let $\mathcal{G}$ be any (possibly empty) set of nonprincipal ideals in $R$. Then

$$
\mathcal{F}:=\mathcal{H} \cup\{h G:(h) \in \mathcal{H}, G \in \mathcal{G}\}
$$

is a $\frac{1}{2}$-monoidal family. Furthermore, any $\frac{1}{2}$-monoidal family in $R$ arises in this way.

Proof. Let $(a), B \in \mathcal{F}$, where $a \in R$ and $B \triangleleft R$. To show that $a B \in \mathcal{F}$, we may assume that $a \neq 0 \neq B$. We must then have $(a) \in \mathcal{H}$. For, if otherwise, $(a)=h G$ for some $(h) \in \mathcal{H}$ and $G \in \mathcal{G}$. This implies that $a=h a_{0}$ for some $a_{0} \in R$. But then $\left(h a_{0}\right)=h G \Rightarrow\left(a_{0}\right)=G \in \mathcal{G}$, a contradiction. If $B \in \mathcal{F}$ is not principal, then $B=h_{1} G_{1}$ for some $\left(h_{1}\right) \in \mathcal{H}$ and $G_{1} \in \mathcal{G}$. In this case, $a B=a h_{1} G_{1} \in \mathcal{F}$, since $\left(a h_{1}\right)=(a)\left(h_{1}\right) \in \mathcal{H}$. Thus, we may assume $B \in \mathcal{F}$ is principal. As before, we see easily that $B \in \mathcal{H}$, so now $a B \in \mathcal{H} \subseteq \mathcal{F}$. This checks that $\mathcal{F}$ is $\frac{1}{2}$-monoidal.

Conversely, let $\mathcal{F}$ be any $\frac{1}{2}$-monoidal family. Define $\mathcal{H}=\{(h):(h) \in \mathcal{F}\}$, and let $\mathcal{G}$ be the set of all nonprincipal ideals in $\mathcal{F}$. Clearly, $\mathcal{H}$ is monoidal, and $\mathcal{F}$ coincides with the $\frac{1}{2}$-monoidal family constructed from $\mathcal{H}$ and $\mathcal{G}$ in the first half of this theorem.

In view of the explicit nature of the construction in (5.5), we may regard the description of Oka families in an integral domain to be "complete" if one can give a satisfactory answer to the following

Question 5.6. When is the $\frac{1}{2}$-monoidal family $\mathcal{F}$ in (5.5) an Oka family? 
Next, we'll show that, analogously to (5.2), strongly Oka families also have "partial" monoidal properties, as follows.

Proposition 5.7. Let $\mathcal{F}$ be a strongly Oka family in a ring $R$, and let $A, B \in \mathcal{F}$.

(1) If $(A B: A)=B$, then $A B \in \mathcal{F}$.

(2) If $A$ is not strongly Oka, then $A^{2} \in \mathcal{F}$.

(3) If $B=\sqrt{B}$ and $A$ is f.g. with ann $(A) \subseteq B$, then $A B \in \mathcal{F}$.

(4) If either $A$ is an invertible ideal or $(A, B)=R$, then $A B \in \mathcal{F}$. Thus, $\mathcal{F}$ is always comaximally monoidal (in the sense of (4.5)).

Proof. (1) The assumption $(A B: A)=B$ implies that $(A B: A) \in \mathcal{F}$. Since $(A B, A)=A \in \mathcal{F}$ also, the strong Oka property yields $A B \in \mathcal{F}$.

(2) If $A$ is not strongly Oka, then $\left(A^{2}: A\right)=A$. Thus, (1) gives $A^{2} \in \mathcal{F}$.

(3) In this case, (3.14) gives $(A B: A) \subseteq \sqrt{B}=B \subseteq(A B: A)$, so (1) applies.

(4) Again, it suffices to check that $B \subseteq(A B: A)$ is an equality. Let $r \in(A B: A)$, so $r A \subseteq A B$. If $A$ is invertible, multiplication by $A^{-1}$ gives $r \in B$. If $A+B=R$ instead, fix an equation $a+b=1$, where $a \in A$ and $b \in B$. Then $r=r a+r b \in$ $A B+B=B$ as well.

Proposition 5.8. Let $\mathcal{F}$ be an ideal family in a noetherian domain $R$ satisfying $(\mathrm{Sq})^{*}$. Then the properties $\left(\mathrm{P}_{2}\right),\left(\mathrm{P}_{3}\right)$, and "Strongly Oka" are equivalent for $\mathcal{F}$.

Proof. Since $\left(\mathrm{P}_{2}\right) \Rightarrow\left(\mathrm{P}_{3}\right) \Rightarrow$ Strongly Oka, it suffices to show that, if $\mathcal{F}$ is strongly Oka, then it is monoidal (for then $\mathcal{F}$ will satisfy $\left(\mathrm{P}_{2}\right)$ ). Let $A, B \in \mathcal{F}$. Since $C:=\sqrt{B}$ is f.g., we have $C^{n} \subseteq B \subseteq C$ for some $n \geq 1$. As $B \in \mathcal{F}$, the property (Sq)* implies that $C \in \mathcal{F}$ (see (2.1)). By (5.7)(3), $A C \in \mathcal{F}$. Replacing $A$ by $A C$ (which is still f.g.), we have $A C^{2} \in \mathcal{F}$, and hence by induction, $A C^{n} \in \mathcal{F}$. But $(A B)^{n}=A^{n} B^{n} \subseteq A C^{n} \subseteq A B$. By (Sq) ${ }^{*}$ again, this implies that $A B \in \mathcal{F}$, as desired.

Without the property $(\mathrm{Sq})^{*}$, however, the situation is not as straightforward as in (5.8), as the three properties in (5.8) are generally different over noetherian domains. We close with an example illustrating the distinction between Oka and strongly Oka families over such domains, followed by an open question.

Example 5.9. Given the work done in [LR], it is not difficult to produce an Oka family in a noetherian domain $R$ that is not strongly Oka. For instance, over $R=k[x, y]$ (for any field $k$ ), let $\mathcal{F}$ be the family of ideals $J \triangleleft R$ such that $\operatorname{dim}_{k} R / J$ is finite and divisible by 3 . Then $\mathcal{F}$ is Oka (by the method of proof for [LR: (5.13)]). However,

(1) $\mathcal{F}$ is not closed under squaring (and hence not monoidal): note that $A:=$ $(x, y)^{2} \in \mathcal{F}\left(\right.$ since $\left.\operatorname{dim}_{k} R / A=3\right)$, but $A^{2} \notin \mathcal{F}\left(\right.$ since $\left.\operatorname{dim}_{k} R / A^{2}=10\right) ;$

(2) the ideal $A \in \mathcal{F}$ above is not strongly Oka (see (3.16)(2)). Since $A^{2} \notin \mathcal{F}$, (5.7)(2) implies that $\mathcal{F}$ is not strongly Oka.

In addition, it can be shown that the ideal $A$ is actually Oka; for more details, see $[\mathrm{KLR}]$. Granted this fact, we will have gotten even a two-member family $\{R, A\}$ (over a noetherian domain $R$ ) that is Oka, but not strongly Oka. 
A significant question to ask in completing the analysis of the dependence chart (2.7) is whether Ako families $\mathcal{F}$ in a ring $R$ are Oka. What makes this question tricky is that its answer happens to be "yes" in a good number of cases; e.g. when $\mathcal{F}$ is a two-member family (by $(3.6)(6)$ ), or when $R$ is a von Neumann regular ring (by $(4.3)(3))$. In the next two sections, we'll show that the answer is also "yes" when $R$ is a Dedekind domain (see (6.2)), or a 1-dimensional valuation domain (see $(7.6)(2))$. Nevertheless, the answer turns out to be "no" in general: (7.10) offers an example of a two-dimensional valuation domain $R$ with an Ako family $\mathcal{F}$ that is not $\frac{1}{2}$-monoidal. By $(5.2), \mathcal{F}$ is not an Oka family. But of course, such a domain $R$ is always non-noetherian. This leaves open the following

Question 5.10. What is an example of a noetherian domain with an Ako family that is not Oka? Or more generally, exactly which rings $R$ are such that every Ako family in $R$ is Oka? (A sufficient condition for such rings was given in (4.3)(1), but we'll see later in (7.7) that this is not a necessary condition.)

\section{Dedekind Domains}

We come now to some special results on Oka and Ako families over Dedekind domains. Such domains share with von Neumann regular rings the property that Ako families in them are Oka (see (6.2)). However, contrary to the case of von Neumann regular rings, it turns out that the adjective "strong" becomes dispensable for such families. To prove these results, we start by handling the Oka case.

Theorem 6.1. A family of ideals $\mathcal{F}$ in a Dedekind domain $R$ is Oka iff it is strongly Oka, iff it is monoidal.

Proof. First assume $\mathcal{F}$ is Oka. To show that $\mathcal{F}$ is monoidal, let $J, K \in \mathcal{F}$, which we may assume to be nonzero. Then $I:=J K \neq 0$. By the " $1 \frac{1}{2}$ generator property" for ideals in a Dedekind domain (see [HL] for the terminology), $J=(I, a)$ for some $a \in R$. Since $J$ is a multiplication ideal, $I=J \cdot(I: J)$. By cancellation, we get $K=$ $(I: J)=(I: a)$. Invoking the Oka property, we have $(I, a),(I: a) \in \mathcal{F} \Rightarrow I \in \mathcal{F}$.

To complete the proof, it suffices to show that, if $\mathcal{F}$ is monoidal, then it is strongly Oka. Let $I, A \triangleleft R$ be such that $(I: A)$ and $J=(I, A)$ both belong to $\mathcal{F}$. Since $J$ is either zero or invertible, and $(I: J)=(I: A) \in \mathcal{F}$, the monoidal property gives $I=J \cdot(I: J) \in \mathcal{F}$, as desired.

Note that the conditions in (6.1) are not equivalent to $\mathcal{F}$ being just $\frac{1}{2}$-monoidal. This is readily shown by the example $\mathcal{F}=\{R, A\}$, where $A$ is a nonprincipal ideal in a Dedekind ring of class number $>1$; see (5.4). In the special case of two-member families, (6.1) shows that (0) is the only Oka (or strongly Oka) ideal in a Dedekind ring $R$. Of course, this conclusion could also have been deduced from Theorems 3.4 and 3.5, using the fact that, for any nonzero ideal $A \subsetneq R$, the conormal $R / A$ module $A / A^{2}$ is actually free of rank 1 .

Next, we turn our attention to Ako families. For Dedekind domains, it is easy to see from $(4.3)(1)$ that Ako families are Oka. In fact, the following result gives even stronger conclusions without explicitly applying (4.3)(1). 
Theorem 6.2. Let $R$ be a Dedekind domain. An ideal family $\mathcal{F}$ in $R$ is Ako iff it is strongly Ako, iff it has the property $\left(\mathrm{P}_{3}\right)$. Any Ako family $\mathcal{F}$ in $R$ is strongly Oka, and is closed under finite products and intersections.

Proof. For the "iff" statements, it suffices to check that "Ako" implies the property (2.4). Let $I, A, B \triangleleft R$ be such that $(I, A),(I, B) \in \mathcal{F}$. To show that $J:=(I, A B) \in \mathcal{F}$, we may clearly assume that $J \neq 0$. Since $J \subseteq(I, A)$, the $1 \frac{1}{2}$-generator property enables us to write $(I, A)=(J, x)$ for some $x$, which we may assume to be in $A$. Similarly, $(I, B)=(J, y)$ for some $y \in B$. Since $\mathcal{F}$ is Ako, $(J, x y) \in \mathcal{F}$. But $(I, A B) \supseteq(J, x y)$ is easily seen to be an equality, so we have $(I, A B) \in \mathcal{F}$, which checks $(2.4)$. The last statement of the theorem follows from (2.3) and (2.7).

What we have done so far in this section is enough for us to "construct" all Oka and Ako families in a Dedekind ring $R$. In the Oka case, (6.1) reduces the problem to that of constructing all monoidal families. This can be done over any ring $R$ by taking any set of ideals in $R$ and forming the family of all finite (including empty) products of such ideals. In the Ako case, (6.2) reduces the problem to that of constructing all $\left(\mathrm{P}_{3}\right)$-families. This can again be done over any ring $R$, as follows. For any $A \triangleleft R$, let

$$
\mathcal{F}_{A}:=\{R\} \cup\left\{J \triangleleft R: A^{n} \subseteq J \subseteq A \text { for some } n \geq 1\right\},
$$

which is clearly the smallest $\left(\mathrm{P}_{3}\right)$-family containing $A$. Now take any ideal family in $R$, and let $\mathcal{A}$ be the family of all finite intersections of ideals in it. Then $\mathcal{F}:=\bigcup_{A \in \mathcal{A}} \mathcal{F}_{A}$ is a $\left(\mathrm{P}_{3}\right)$-family. First, $\mathcal{F}$ has the property $(\mathrm{Sq})$ (since each $\mathcal{F}_{A}$ does), so it suffices to show that $C, D \in \mathcal{F} \Rightarrow C \cap D \in \mathcal{F}$. Say $C \in \mathcal{F}_{A}$, and $D \in \mathcal{F}_{B}$, where $A, B \in \mathcal{A}$. For a large $n$, we have $A^{n} \subseteq C \subseteq A$, and $B^{n} \subseteq D \subseteq B$. Thus, for $E:=A \cap B \in \mathcal{A}$, we have $E^{n} \subseteq C \cap D \subseteq E$, so $C \cap D \in \mathcal{F}_{E} \subseteq \mathcal{F}$. This checks that $\mathcal{F}$ is a $\left(\mathrm{P}_{3}\right)$-family. Conversely, any $\left(\mathrm{P}_{3}\right)$-family $\mathcal{F}$ arises in this way, by taking $\mathcal{A}$ to be $\mathcal{F}$ in the above construction.

Remark 6.4. While Ako families are Oka in Dedekind rings (by (6.2)), the constructions above showed quite clearly that the converse is not true - even for a discrete valuation ring $(R, \mathfrak{m})$. In such a ring, for instance, the (strongly) Oka family $\mathcal{F}=\left\{\mathfrak{m}^{2 i}: i \geqslant 0\right\}$ is not Ako, since the (Sq) property obviously fails. ${ }^{3}$ Indeed, by the construction method given in the last paragraph, we see easily that the Ako families in $(R, \mathfrak{m})$ are precisely: $\mathcal{F}_{\mathfrak{m}^{i}}$ and $\mathcal{F}_{\mathfrak{m}^{i}} \cup\{(0)\}$, where $i \geqslant 0$.

Question 6.5. To what extent do the results in this section hold for Prüfer domains and 1-dimensional noetherian domains?

\section{Valuation Domains}

The class of valuation domains happens to be a fertile ground on which to study the Oka and Ako properties (and their strong analogues). In fact, we will be able to determine rather precisely all ideal families in valuation domains that have, respectively, the Oka, Ako, or $\left(\mathrm{P}_{3}\right)$ properties. These determination criteria, in particular, enable us to construct in (7.8) the first example of an Ako family that

\footnotetext{
${ }^{3}$ For some earlier examples of strongly Oka families that are not Ako, see [LR: (3.14), (3.18)].
} 
is not Oka - in a 2-dimensional valuation domain. It turns out, nevertheless, that in any 1-dimensional valuation domain, Ako families are Oka (see (7.6)(2)).

We start with a result (7.1) that characterizes Oka families in valuation domains. In view of this result, (5.4) gives the construction of all Oka families in such domains.

Theorem 7.1. An ideal family $\mathcal{F}$ in a valuation domain $R$ is Oka iff it is $\frac{1}{2}$-monoidal. A proper ideal $A \triangleleft R$ is Oka iff $A=(0)$ or $A$ is not principal.

Proof. The "only if" part is true for all domains, by (5.2). For the "if" part, suppose $\mathcal{F}$ is $\frac{1}{2}$-monoidal. Let $a \in R$ and $I \triangleleft R$ be such that $(I, a)$ and $(I: a)$ both belong to $\mathcal{F}$. If $a \in I$, then $I=(I, a) \in \mathcal{F}$ already. If $a \notin I$, then $I \subseteq(a)$ (since $R$ is a valuation domain). But then $(a)=(I, a) \in \mathcal{F}$, and $\mathcal{F}$ being $\frac{1}{2}$-monoidal implies $I=a \cdot(I: a) \in \mathcal{F}$. This shows that $\mathcal{F}$ is Oka. The last conclusion now follows from (3.6)(4) since 0,1 are the only idempotents in $R$.

We now turn our attention to Ako families. According to (7.1), to show that "Ako $\nRightarrow$ Oka" in general, it would suffice to come up with an Ako family in some integral domain that is not $\frac{1}{2}$-monoidal. Searching for such examples over valuation domains led us to the following characterization for Ako families over them. (Note that (3), $(3)^{\prime}$ below are special cases of the squeezing property (Sq), although their equivalence would not follow from the proof given earlier in (2.1).)

Theorem 7.2. For an ideal family $\mathcal{F}$ in a valuation domain $R$, the following are equivalent (where, as usual, $b \in R$ and $J \triangleleft R$ are arbitrary):

(1) $\mathcal{F}$ is an Ako family.

(2) $(J, b) \in \mathcal{F} \Longrightarrow\left(J, b^{2}\right) \in \mathcal{F}$.

$(2)^{\prime}(J, b) \in \mathcal{F} \Longrightarrow\left(J, b^{n}\right) \in \mathcal{F}$ for every $n \geq 2$.

(3) $(b)^{2} \subseteq J \subseteq(b) \in \mathcal{F} \Longrightarrow J \in \mathcal{F}$.

$(3)^{\prime}(b)^{n} \subseteq J \subseteq(b) \in \mathcal{F}$ (for some $\left.n \geq 2\right) \Longrightarrow J \in \mathcal{F}$.

(4) For any $(a),(b) \in \mathcal{F},(a b) \subseteq J \subseteq(a) \cap(b) \Longrightarrow J \in \mathcal{F}$.

Any union of Ako families in a valuation domain $R$ is an Ako family. A proper ideal $A \triangleleft R$ is Ako iff it is Oka; that is, iff $A=0$ or $A$ is nonprincipal.

Proof. The conclusion about unions is clear from any one of the characterizations $(2),(2)^{\prime},(3)$ and $(3)^{\prime}$ (but not (4)!). In the same spirit, the description of the Ako ideals follows easily from the characterization (4). To prove the equivalence of the six conditions, first note that $(1) \Rightarrow(2)$ is trivial.

$(2) \Rightarrow(3)$. Assume $(b)^{2} \subseteq J \subseteq(b) \in \mathcal{F}$. Since $(J, b)=(b) \in \mathcal{F},(2)$ implies that $\left(J, b^{2}\right) \in \mathcal{F}$. But $b^{2} \in J$, so we have $J \in \mathcal{F}$. A similar argument gives $(2)^{\prime} \Rightarrow(3)^{\prime}$. (So far, $R$ could have been any ring.)

$(3) \Rightarrow(4)$. Given $(a),(b) \in \mathcal{F}$ and $(a b) \subseteq J \subseteq(a) \cap(b)$, we may assume by symmetry that $(b) \subseteq(a)$. Then $(b)^{2} \subseteq(a b) \subseteq J \subseteq(b)$, so (3) gives $J \in \mathcal{F}$.

$(4) \Rightarrow(1)$. Given $(I, a),(I, b) \in \mathcal{F}$, we would like to show that $(I, a b) \in \mathcal{F}$. We may assume that $a, b \notin I$. Since $R$ is a valuation domain, we then have $I \subseteq(a) \cap(b)$, in which case our hypothesis gives $(a),(b) \in \mathcal{F}$. But now $(a b) \subseteq(I, a b) \subseteq(a) \cap(b)$, so (4) gives $(I, a b) \in \mathcal{F}$. 
The proof is completed by noting that $(1) \Rightarrow(2)^{\prime} \Rightarrow(3)^{\prime} \Rightarrow(3)$.

A similar analysis shows that a family $\mathcal{F}$ on a valuation domain is strongly Ako iff, for $(a), B \in \mathcal{F}, a B \subseteq I \subseteq(a) \cap B \Rightarrow I \in \mathcal{F}$. However, this does not imply that the union of strongly Ako families is strongly Ako. The analogy (with (7.2)) works much better with the property $\left(\mathrm{P}_{3}\right)$, for which we have the following result.

Theorem 7.3. For an ideal family $\mathcal{F}$ in a valuation domain $R$, the following are equivalent (where, as usual, $B, J \triangleleft R$ are arbitrary):

(1) $\mathcal{F}$ has the property $\left(\mathrm{P}_{3}\right)$.

(2) $(J, B) \in \mathcal{F} \Longrightarrow\left(J, B^{2}\right) \in \mathcal{F}$.

$(2)^{\prime}(J, B) \in \mathcal{F} \Longrightarrow\left(J, B^{n}\right) \in \mathcal{F}$ for every $n \geq 2$.

(3) $\mathcal{F}$ has the property $(\mathrm{Sq})$.

Any union of $\left(\mathrm{P}_{3}\right)$ families in a valuation domain $R$ is a $\left(\mathrm{P}_{3}\right)$ family.

Proof. This can be shown exactly as in the proof of (7.2), with the principal ideal (b) replaced everywhere by an arbitrary ideal $B$. (Of course, $(1) \Leftrightarrow(3)$ is already clear since any family $\mathcal{F}$ in $R$ is closed under finite intersections.)

Remark 7.4. In the paragraph following the proof of (6.2), we have given the general construction of $\left(\mathrm{P}_{3}\right)$-families in all rings. Since any ideal family in a valuation ring $R$ is closed under finite intersections (as we have just observed above), this construction simplifies further to the following:

- The $\left(\mathrm{P}_{3}\right)$-families in $R$ are exactly those of the form $\mathcal{F}:=\bigcup_{A \in \mathcal{A}} \mathcal{F}_{A}$,

where $\mathcal{A}$ is any set of ideals in $R$, and $\mathcal{F}_{A}$ (for any ideal $A \triangleleft R$ ) is defined to be $\{R\} \cup\left\{J \triangleleft R: A^{n} \subseteq J \subseteq A\right.$ for some $\left.n \geq 1\right\}$, as in (6.3).

In the case where $A$ is a principal ideal $(a)$, we'll write the family $\mathcal{F}_{A}$ more simply as $\mathcal{F}_{a}$. Using this notation, and proceeding in the same manner as in the Oka case, we can now construct all Ako families in a valuation domain $R$ as follows.

Theorem 7.5. Let $S$ be a subset of a valuation domain $R$ with $1 \in S$, and let $\mathcal{G}$ be any (possibly empty) family of nonprincipal ideals in $R$. Then $\mathcal{F}:=\mathcal{G} \cup \bigcup_{a \in S} \mathcal{F}_{a}$ is an Ako family of $R$. Furthermore, every Ako family in $R$ arises in this way.

Proof. We'll check that $\mathcal{F}$ has the property $(2)^{\prime}$ in $(7.2)$. Say $(b)^{n} \subseteq J \subseteq(b) \in \mathcal{F}$, where $b \in R$ and $J \triangleleft R$. Since $(b) \notin \mathcal{G}$, we have $(b) \in \mathcal{F}_{a}$ for some $a \in S$. Recalling that $\mathcal{F}_{a}$ has the property $\left(\mathrm{P}_{3}\right)$ (and hence the property $(\mathrm{Sq})$ ), we see that $J \in \mathcal{F}_{a} \subseteq \mathcal{F}$.

Conversely, let $\mathcal{F}$ be any Ako family in $R$. Consider any principal ideal $(a) \in \mathcal{F}$. By (7.2), any ideal between $(a)$ and some $(a)^{n}$ is in $\mathcal{F}$; that is, $\mathcal{F}_{a} \subseteq \mathcal{F}$. Thus, $\mathcal{F}$ is a union of all such $\mathcal{F}_{a}$ 's, and all of the nonprincipal ideals in $\mathcal{F}$.

For 1-dimensional valuation domains $R,(1)$ of the following result gives a very explicit computation of the families $\mathcal{F}_{A}$ for any $A \triangleleft R$. This leads once more to the special implication "Ako $\Rightarrow$ Oka" for $R$, which has been proved before for Dedekind domains and for von Neumann regular rings by different methods (in (6.2) and (4.3)(3) respectively). 
Theorem 7.6. Let $(R, \mathfrak{m})$ be a 1-dimensional valuation domain, and let $A \triangleleft R$.

(1) If $A=(0), R$, or if $A=\mathfrak{m}$ and $R$ is non-noetherian, then $\mathcal{F}_{A}=\{R, A\}$. Otherwise, $\mathcal{F}_{A}=\{R\} \cup\{J \triangleleft R: 0 \neq J \subseteq A\}$.

(2) Any Ako family $\mathcal{F}$ in $R$ is Oka.

Proof. (1) The cases $A=(0), R$ are trivial. If $A=\mathfrak{m}$ and $R$ is non-noetherian, we have $\mathfrak{m}=\mathfrak{m}^{2}$. (Otherwise, $\mathfrak{m}$ would have to be principal by an easy argument, in which case $R$ would be a DVR.) Thus, $\mathcal{F}_{A}=\{R, \mathfrak{m}\}=\{R, A\}$. Now assume we are not in any of the cases above. If $A=\mathfrak{m}, R$ is then noetherian (and hence a DVR), in which case $\mathcal{F}_{A}$ is certainly the set of all nonzero ideals in $A$. Finally, assume $A \subsetneq \mathfrak{m}$. It is well known that $\mathfrak{p}:=\bigcap_{n \geqslant 1} A^{n}$ is a prime ideal, so it is (0) (since $\mathfrak{p} \subsetneq \mathfrak{m}$ and $\operatorname{dim} R=1$ ). For any nonzero subideal $J \subseteq A$, we have then $J \nsubseteq A^{n}$ for some $n \geq 1$. Thus, $A^{n} \subseteq J$, and so $J \in \mathcal{F}_{A}$.

(2) Express the Ako family $\mathcal{F}$ in the form $\mathcal{G} \cup \bigcup_{a \in S} \mathcal{F}_{a}$ as in (7.5). To show that $\mathcal{F}$ is Oka, it suffices (by (7.1)) to check that $\mathcal{F}$ is $\frac{1}{2}$-monoidal; that is, $(c), B \in \mathcal{F} \Rightarrow c B \in \mathcal{F}$. We may (of course) assume that $B \neq(0)$. Since $(c) \notin \mathcal{G}$, we have $(c) \in \mathcal{F}_{a}$ for some $a \in S$. If $a \notin \mathfrak{m}$, then $(c)=R$, so $c B=B \in \mathcal{F}$. Now assume $a \in \mathfrak{m}$. We may also assume $a \neq 0$ (for otherwise $c=0$ and there is nothing to prove). If $(a) \subsetneq \mathfrak{m}$, part (1) implies that

$$
\mathcal{F}_{a}=\{R\} \cup\{J \triangleleft R: 0 \neq J \subseteq(a)\} .
$$

Since $0 \neq c B \subseteq(a)$, we have $c B \in \mathcal{F}_{a} \subseteq \mathcal{F}$. Thus, we are down to the case where $(a)=\mathfrak{m}$. In this case, $R$ is a DVR. Here, $0 \neq c B \subseteq(a)$ again implies that $c B \in \mathcal{F}_{a} \subseteq \mathcal{F}$. (Having proved (2), it is appropriate to recall that its "strong analogue" is false: in the proof of $(3.10)$, we have already seen that "Str. Ako $\nRightarrow$ Str. Oka" for 1-dimensional valuation domains!)

Example 7.7. Since we did have a sufficient condition in (4.3)(1) for all Ako families in a given ring to be Oka, it is natural to ask if this condition can be applied to 1-dimensional valuation rings to give the conclusion (7.6)(2). Unfortunately, it cannot. In fact, the 1-dimensional valuation ring $R$ associated with a rank 1 valuation $v: K \rightarrow \mathbb{Q} \cup\{\infty\}$ does not satisfy the condition (4.3)(1). To see this, consider the ideal $A \triangleleft R$ defined in (3.12), and fix an element $a \in R$ such that $v(a)=1$. Then

$$
(A: a)=\{r \in R: v(r)>\sqrt{2}-1\} \supsetneq A .
$$

If $(A: a)=(A, b)$ for some $b \in R$, then $b \notin A$ by the above, and so $A \subseteq(b)$. But then $(A: a)=(b)$, which contradicts the description of $(A: a)$ in $(7.8)$.

In view of (7.6)(2), to construct an Ako family in a valuation domain that is not Oka requires working with valuation domains of dimension $\geqslant 2$. Our construction will be based on the statement on unions of Ako families in (7.2). Indeed, the main idea of the next lemma is exactly that, while the union of two Ako families in a valuation domain is always Ako, there is a substantial chance that it may not be Oka. Of course, a part of the "intuition" behind this lemma also came from the proof of Theorem 7.6 above. 
Lemma 7.9. Let $B$ be a nonprincipal and nonprimary ideal in a valuation domain $R$; say $a, a^{\prime} \in R$ are such that $a^{\prime} \notin B, a \notin \sqrt{B}$, but $a a^{\prime} \in B$. Then $\mathcal{F}=\{B\} \cup \mathcal{F}_{a}$ is an Ako family, but not an Oka family.

Proof. That $\mathcal{F}$ is Ako follows from (7.5). Assume for now that $\mathcal{F}$ is Oka. Then $(a) \in \mathcal{F}_{a} \subseteq \mathcal{F}$ and $B \in \mathcal{F}$ imply that $a B \in \mathcal{F}$ by (5.2). Now $a B \subsetneq B$, since $a a^{\prime} \in B \backslash a B$. Therefore, we must have $a B \in \mathcal{F}_{a}$; that is, $a B \supseteq(a)^{n}$ for some $n \geq 1$. But then $a^{n-1} \in B$, a contradiction.

Example 7.10. To get an explicit example of an Ako family that is not Oka, we need only produce a valuation ring $R$ with the data in (7.9). Let $\Gamma$ be the additive group $\mathbb{Z} \oplus \mathbb{Q}$, ordered lexicographically with respect to the usual orderings on $\mathbb{Z}$ and $\mathbb{Q}$. Take any field $K$ with a Krull valuation $v: K \rightarrow \Gamma \cup\{\infty\}$ (for the existence of $K$, see, again, [Ri:p.368]), and let $R$ be the corresponding (2-dimensional) valuation ring in $K$. Let $B$ be the ideal $\{x \in R: v(x)>(1,0)\}$. This is a nonprincipal ideal, since $\{\alpha \in \Gamma: \alpha>(1,0)\}$ does not have a smallest element in the value group $\Gamma$. Consider elements $a, a^{\prime} \in R$ such that $v(a)=(0,2)$ and $v\left(a^{\prime}\right)=(1,-1)$. Clearly, $a^{\prime} \notin B$, and since $v\left(a^{n}\right)=(0,2 n)$, we have $a^{n} \notin B$ for all $n \geq 1$. Finally,

$$
v\left(a a^{\prime}\right)=(0,2)+(1,-1)=(1,1)>(1,0)
$$

shows that $a a^{\prime} \in B$. Thus, $B$ is nonprimary, nonprincipal, and we have realized the full set of data in (7.9).

Theorem 7.12. The logical dependence chart (2.7) is the best possible for valuation domains, in that no more implication arrows can be added to the chart - other than those that can obtained by compositions.

Proof. The proof of (3.10) already showed that, for valuation domains, "Str. Ako $\nRightarrow$ Str. Oka", and (6.4) showed that "Str. Oka $\nRightarrow$ (Str.) Ako". These non-implications imply, as in the proof of (3.10), that the four legs in the square part of (2.7) are each irreversible for valuation domains. With the new Example 7.10, we have "Ako $\nRightarrow$ Oka", so we get for free "Ako $\nRightarrow$ Strongly Ako" as well. (Recall, however, that the last two non-implications cannot be proved by using two-member families over any ring!) Finally, $\left(\mathrm{P}_{2}\right) \nRightarrow\left(\mathrm{P}_{1}\right)$ and $\left(\mathrm{P}_{3}\right) \nRightarrow\left(\mathrm{P}_{2}\right)$ can be shown by fixing a $\operatorname{DVR}(R, \mathfrak{m})$, and taking, respectively, $\mathcal{F}=\{R,(0)\}$ and $\mathcal{F}=\left\{\mathfrak{m}^{i}: i \neq 1\right\}$.

\section{References}

$\left[\mathrm{An}_{1}\right]$ D. D. Anderson: Multiplication ideals, multiplication rings, and the ring $R(X)$. Canad. J. Math. 29 (1976), 760-768.

$\left[\mathrm{An}_{2}\right]$ D. D. Anderson: Some remarks on multiplication ideals. Math. Japon. 25 (1980), 463-469.

[Ba] H. Bass: Finitistic dimension and a homological generalization of semiprimary rings. Trans. A.M.S. 95 (1960), 466-488.

[BG] J. W. Brewer, S. Glaz, W. Heinzer and B. Olberding (eds.): Multiplicative Ideal Theory in Commutative Algebra. A Tribute to the Work of Robert Gilmer. Springer-Verlag, BerlinHeidelberg-New York, 2006.

[Co] I. S. Cohen: Commutative rings with restricted chain condition. Duke Math. J. 17 (1950), $27-42$.

[Gi] R. Gilmer: Multiplicative Ideal Theory. Queen's Papers in Pure and Applied Mathematics, Vol. 90, Queen's University, Kingston, Ontario, Canada, 1992. 
[GM] R. Gilmer and J.L. Mott: Multiplication rings in which ideals with prime radicals are primary. Trans. A.M.S. 114 (1965), 40-52.

[HK] F. Halter-Koch: Ideal Systems. An introduction to Multiplicative Ideal Theory. Mono. Pure \& Applied Math., Vol. 211, M. Dekker, Inc., New York, 1998.

[HL] R. C. Heitmann and L.S. Levy: $1 \frac{1}{2}$ and 2 generator ideals in Prüfer domains. Rocky Mountain J. Math. 5 (1975), 361-373.

[Ja] P. Jaffard: Les Systèmes d'Idéaux. Travaux et Recherches Mathématiques, Vol. IV, Dunod, Paris, 1960.

[Ka] I. Kaplansky: Commutative Rings. Revised Ed., Univ. Chicago Press, 1974.

$[\mathrm{KLR}]$ V. Kodiyalam, T. Y. Lam and M. L. Reyes: note in preparation.

[Kr] W. Krull: Idealtheorie. Ergeb. Math. 4, Springer-Verlag, Berlin, 1935.

$[\mathrm{Ku}]$ E. Kunz: Introduction to Commutative Algebra and Algebraic Geometry. Birkhäuser, Boston-Basel-Stuttgart, 1985.

[La $]$ T. Y. Lam: Lectures on Modules and Rings. Graduate Texts in Math., Vol. 189, SpringerVerlag, Berlin-Heidelberg-New York, 1999.

[La2] T. Y. Lam: Exercises in Classical Ring Theory. Second Edition, Problem Books in Math., Springer-Verlag, Berlin-Heidelberg-New York, 2001.

[La3] T. Y. Lam: Serre's Problem on Projective Modules. Monographs in Math., Springer-Verlag, Berlin-Heidelberg-New York, 2006.

[LR] T. Y. Lam and M. L. Reyes: A prime ideal principle in commutative algebra. J. Algebra 319 (2008), 3006-3027.

[LM] M. D. Larsen and P. J. McCarthy: Multiplicative Theory of Ideals. Pure and Applied Math., Vol. 43, Academic Press, New York-London, 1971.

[Lo] P. Lorenzen: Abstrakte Begründung der multiplikativen Idealtheorie. Math. Zeit. 45 (1939). 533-553.

[Mo] J. L. Mott: Equivalent conditions for a ring to be a multiplication ring. Canad. J. Math. 16 (1964), 429-434.

[Na] M. Nagata: Local Rings. John Wiley \& Sons, Inc., New York, 1962.

[Ni] W. K. Nicholson: Lifting idempotents and exchange rings. Trans. A.M.S. 229 (1977), 269278.

[Ok] K. Oka: Sur les fonctions analytiques de plusieurs variables, VIII. J. Math. Soc. Japan 3 (1951), 204-214.

[Ri] P. Ribenboim: The Theory of Classical Valuations. Monographs in Mathematics, SpringerVerlag, Berlin-Heidelberg-New York, 1999.

[Wa] R. B. Warfield: Exchange rings and decompositions of modules. Math. Ann. 199 (1972), $32-36$.

Department of Mathematics, University of California, Berkeley, California 94720

E-mail address: lam@math.berkeley.edu

Department of Mathematics, University of California, Berkeley, California 94720

E-mail address: mreyes@math. berkeley.edu 